\title{
BIN2 orchestrates platelet calcium signaling in thrombosis and thrombo-inflammation
}

\author{
Julia Volz,,$^{1,2}$ Charly Kusch, ${ }^{1,2}$ Sarah Beck, ${ }^{1,2}$ Michael Popp, ${ }^{1,2}$ Timo Vögtle, ${ }^{1,2}$ Mara Meub, ${ }^{3}$ Inga Scheller, ${ }^{1,2}$ Hannah S. Heil, ${ }^{2}$ \\ Julia Preu, ${ }^{1,2}$ Michael K. Schuhmann, ${ }^{4}$ Katherina Hemmen, ${ }^{2}$ Thomas Premsler, ${ }^{5}$ Albert Sickmann, ${ }^{5,6,7}$ Katrin G. Heinze, ${ }^{2}$ \\ David Stegner, ${ }^{1,2}$ Guido Stoll, ${ }^{4}$ Attila Braun, ${ }^{1,2}$ Markus Sauer, ${ }^{3}$ and Bernhard Nieswandt ${ }^{1,2}$ \\ IInstitute of Experimental Biomedicine I, University Hospital Würzburg, Würzburg, Germany. ${ }^{2}$ Rudolf Virchow Center, University of Würzburg, Würzburg, Cermany. ${ }^{3}$ Department of Biotechnology and Biophysics, \\ Biocenter, University of Würzburg, Würzburg, Germany. ${ }^{4}$ Department of Neurology, University Hospital Würzburg, Würzburg, Germany. ${ }^{5}$ Leibniz-Institut für Analytische Wissenschaften, Dortmund, Germany. \\ ${ }^{6}$ Medizinisches Proteom-Center, Ruhr-University Bochum, Bochum, Germany. ${ }^{7}$ Department of Chemistry, College of Physical Sciences, University of Aberdeen, Aberdeen, United Kingdom.
}

\begin{abstract}
Store-operated $\mathrm{Ca}^{2+}$ entry (SOCE) is the major route of $\mathrm{Ca}^{2+}$ influx in platelets. The $\mathrm{Ca}^{2+}$ sensor stromal interaction molecule 1 (STIM1) triggers SOCE by forming punctate structures with the $\mathrm{Ca}^{2+}$ channel Orai1 and the inositol trisphosphate receptor $\left(I P_{3} R\right)$, thereby linking the endo-/sarcoplasmic reticulum to the plasma membrane. Here, we identified the BAR domain superfamily member bridging integrator 2 (BIN2) as an interaction partner of STIM1 and IP $R$ in platelets. Deletion of platelet BIN2 (Bin $2^{f / f f l, P f 4-C r e}$ mice) resulted in reduced $\mathrm{Ca}^{2+}$ store release and $\mathrm{Ca}^{2+}$ influx in response to all tested platelet agonists. These defects were a consequence of impaired IP $R$ function in combination with defective STIM1-mediated SOC channel activation, while $\mathrm{Ca}^{2+}$ store content and agonist-induced $\mathrm{IP}_{3}$ production were unaltered. This severely defective $\mathrm{Ca}^{2+}$ signaling translated into impaired thrombus formation under flow and a protection of Bin $2^{\text {flffl, } f 4-\text { Cre }}$ mice in models of arterial thrombosis and stroke. Our results establish BIN2 as a central regulator of platelet activation in thrombosis and thrombo-inflammatory disease settings.
\end{abstract}

\section{Introduction}

Platelet activation and aggregation at the injured vessel wall is essential for primary hemostasis, but may also trigger occlusion of diseased vessels, resulting in acute ischemia and infarction of vital organs (1). Platelet inhibition is therefore a central strategy for prevention and treatment of myocardial infarction (2) and stroke $(3,4)$. Platelet activation is triggered by exposed constituents of the vessel wall or surrounding tissue, such as collagens and podoplanin, respectively, or soluble mediators such as adenosine diphosphate (ADP), thromboxane $\mathrm{A}_{2}$, and thrombin locally generated by the coagulation cascade (5). These agonists act through 2 major classes of signaling receptors, namely immunoreceptor tyrosine-based activation motif-coupled (ITAM-coupled) receptors or G protein-coupled receptors (GPCRs). While these receptors trigger different signaling pathways, they all activate phospholipase (PL) C isoforms, and thus production of diacylglycerol (DAG) and inositol 1,4,5-trisphosphate (IP $\left.{ }_{3}\right) . \mathrm{IP}_{3}$ binds to inositol trisphosphate receptors $\left(\mathrm{IP}_{3} \mathrm{R}\right)$, thereby inducing the release of $\mathrm{Ca}^{2+}$ from the dense tubular system (DTS), which is the platelet equivalent of the ER. This store depletion triggers a large $\mathrm{Ca}^{2+}$ influx across the plasma membrane $(\mathrm{PM})$, called store-operated $\mathrm{Ca}^{2+}$ entry (SOCE), which is the major route of $\mathrm{Ca}^{2+}$ influx in plate-

Authorship note: JV and CK contributed equally to this work Conflict of interest: The authors have declared that no conflict of interest exists. Copyright: ( 2020, American Society for Clinical Investigation.

Submitted: January 14, 2020; Accepted: July 31, 2020; Published: October 12, 2020.

Reference information: J Clin Invest. 2020;130(11):6064-6079.

https://doi.org/10.1172/JCl136457. lets $(5,6)$. Stromal interaction molecule 1 (STIM1) has been identified as the essential $\mathrm{Ca}^{2+}$ sensor localized in the DTS membrane, regulating the $\mathrm{Ca}^{2+}$ release-activated $\mathrm{Ca}^{2+}$ (CRAC) channel Orai1 in the PM of platelets (7-10).

The functional coupling model, developed in cells other than platelets, suggests that STIM1, Orai1, and IP $\mathrm{R}_{3}$ form punctate structures at the ER-PM junctions, where the actual SOCE may then occur (11-17). While this process forms the essential framework for SOCE in virtually all nonexcitable cells, several enzymes and adaptor proteins have been identified to be critical regulators of this process (17-20), thereby allowing a cell-specific assembly of $\mathrm{Ca}^{2+}$ signaling systems with very different spatial and temporal dynamics $(21,22)$. To date, STIM1 adaptors in platelets remain to be identified.

BIN proteins constitute a family of adapter proteins characterized by a common N-terminal Bin-amphiphysin-Rvs (BAR) domain. Diverse cellular functions of this protein family have been described, including membrane dynamics (23), organization of the actin cytoskeleton (24), cell cycle control (25), and tumor suppression $(25,26)$. Three different BIN isoforms are known: BIN1, BIN2, and BIN3. BIN1 is ubiquitously expressed and has been shown to fold cardiac T-tubules, thereby facilitating cytoskeleton-based $\mathrm{Ca}^{2+}$ channel trafficking to the T-tubule membranes and restraining free diffusion of local $\mathrm{Ca}^{2+}$ and $\mathrm{K}^{+}$ions $(27,28)$. The role of BIN3 in cellular processes is barely understood, except for its involvement in the regulation of Rac1- and Cdc42-dependent processes in myogenesis (29). Likewise, only very limited information about the function of BIN2, predominantly found in hematopoietic cells (30), exists. Previous studies showed that BIN2 is present at podosomes of adherent leuko- 
A

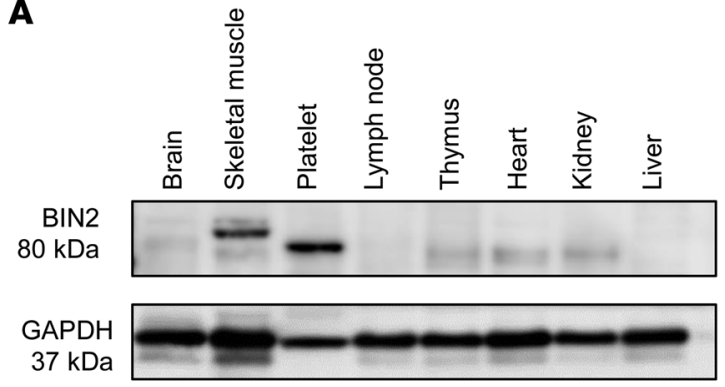

C

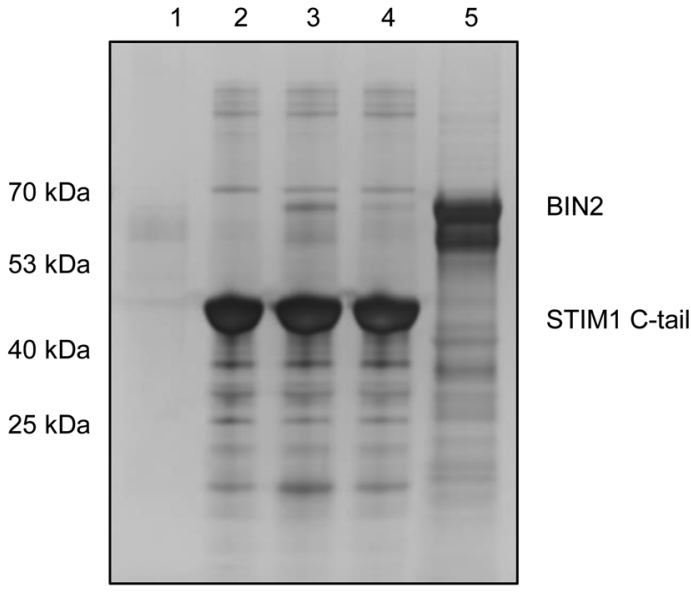

1: rec. Bin2 $(2.5 \mu \mathrm{M})$

2: rec. STIM1 $(2.5 \mu \mathrm{M})$

3: rec. STIM1 $(2.5 \mu \mathrm{M})+$ rec. BIN2 $(125 \mu \mathrm{M})(1: 50)$

4: rec. STIM1 $(2.5 \mu \mathrm{M})+$ rec. BIN2 $(2.5 \mu \mathrm{M})(1: 1)$

5: rec. Bin2 input loaded on Gel
B

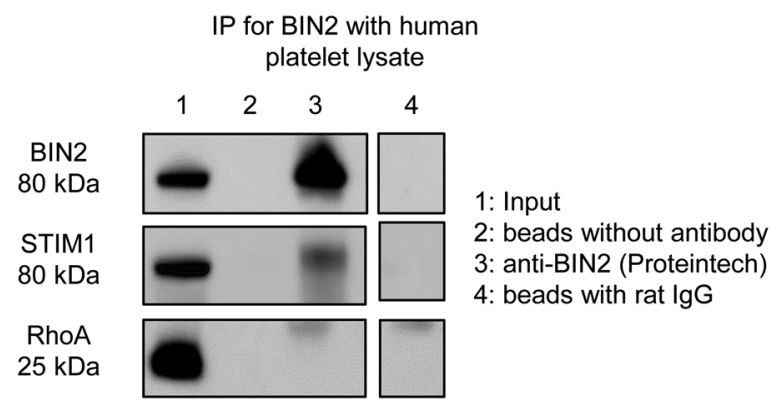

D

WT

Bin2-

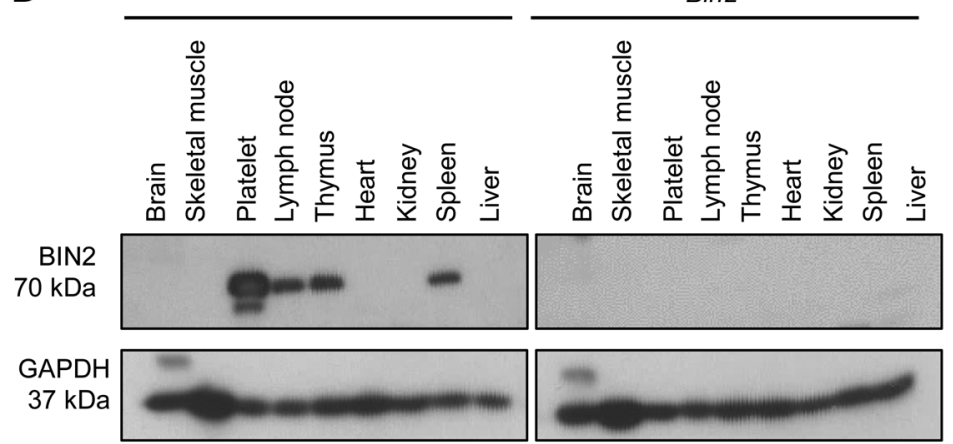

Figure 1. BIN2 is highly expressed in human and mouse platelets. (A) Western blot analysis of BIN2 expression in human tissue lysates; GAPDH was used as loading control. (B) IP of BIN2 was performed with human platelet lysates using an anti-BIN2 antibody. BIN2, STIM1, and RhoA were detected by Western blot. Representative result of 3 independent experiments. Lane 4 was run on the same gel as 1 to 3 , but the lanes were noncontiguous. (C) Recombinant CST-tagged STIM1 C-tail incubated with recombinant BIN2 protein. Pulldown of the GST-tagged STIM1 was performed, and the resulting fractions were analyzed by SDS page and silver staining. (D) Western blot analysis of BIN2 expression in the indicated tissue lysates of WT and Bin2 ${ }^{-/-}$mice with GAPDH as loading control. See complete unedited blots in the supplemental material.

cytes, indicating a functional role in cell adhesion and possibly integrin activation (31). In addition, siRNA knockdown of BIN2 in macrophages leads to enhanced phagocytosis and reduced cell movement of monocytes and mast cells (31).

Here, we used biochemical and proteomic approaches in combination with super-resolution microscopy to identify BIN2 as a highly expressed interaction partner of STIM1 in platelets. Using a megakaryocyte/platelet-specific KO mouse model, we showed that BIN2 plays a key role in $\mathrm{Ca}^{2+}$ store release and SOCE in platelets by directly or indirectly interacting with 2 key molecules in $\mathrm{Ca}^{2+}$ homeostasis, $\mathrm{IP}_{3} \mathrm{R}$ and STIM1. This selective $\mathrm{Ca}^{2+}$ signaling defect translates into protection of Bin $2^{f / f h, P f-C r e}$ mice in models of arterial thrombosis and stroke.

\section{Results}

STIM1 interacts with BIN2 in platelets. To identify STIM1 binding partners in platelets, we coupled the recombinantly expressed C-tail of human STIM1 (aa 484-685; Supplemental Figure 1A; supplemental material available online with this article; https://
doi.org/10.1172/JCI136457DS1) to an Affi-Gel 10 matrix affinity column and loaded it with Triton X-100-lysed resting human platelets (10 mg per $\mathrm{mL}$ affinity support). Loading of equal volumes of pooled platelet lysates on uncoupled Affi-Gel 10 matrix (without the STIM1 C-tail) served as a negative control. Eluted fractions of the affinity columns were resolved by 1D SDS-PAGE, and bands were visualized by subsequent silver staining (Supplemental Figure 1B). Eighteen differential protein bands that were reproducibly detected only in the STIM1 C-tail affinity column samples were excised, tryptically digested, analyzed by nano-LCESI-MS/MS, and compared against the UniProtKB/Swiss-Prot human subset. With this approach, we identified more than 25 STIM1 C-tail bound proteins, among them several cytoskeletal proteins (Supplemental Table 1). After analysis of different hits, we took special interest in the robustly detectable adapter protein bridging integrator 2 (BIN2) (UniProtKB/Swiss-Prot accession number: Q9UBW5-1), given the involvement of BIN1 in $\mathrm{Ca}^{2+}$ signaling of cardiomyocytes. In line with previous reports (30), immunoblotting of different human tissues confirmed the 
A

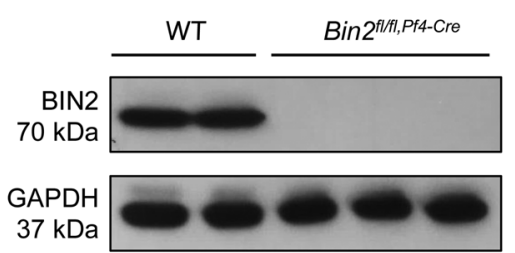

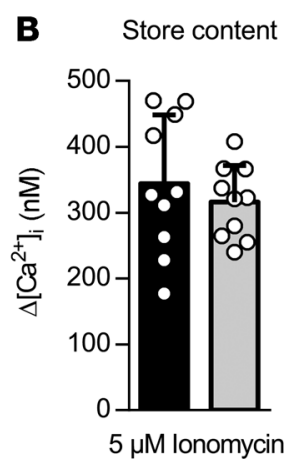

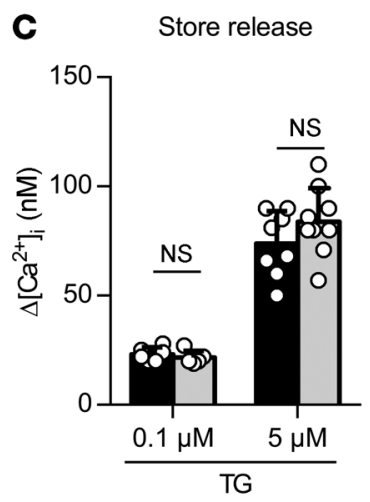

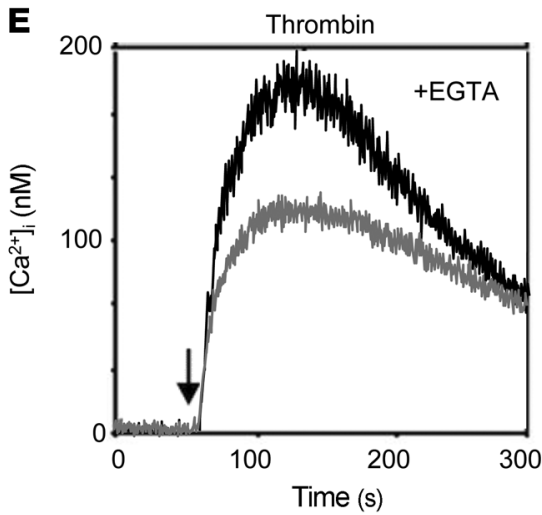
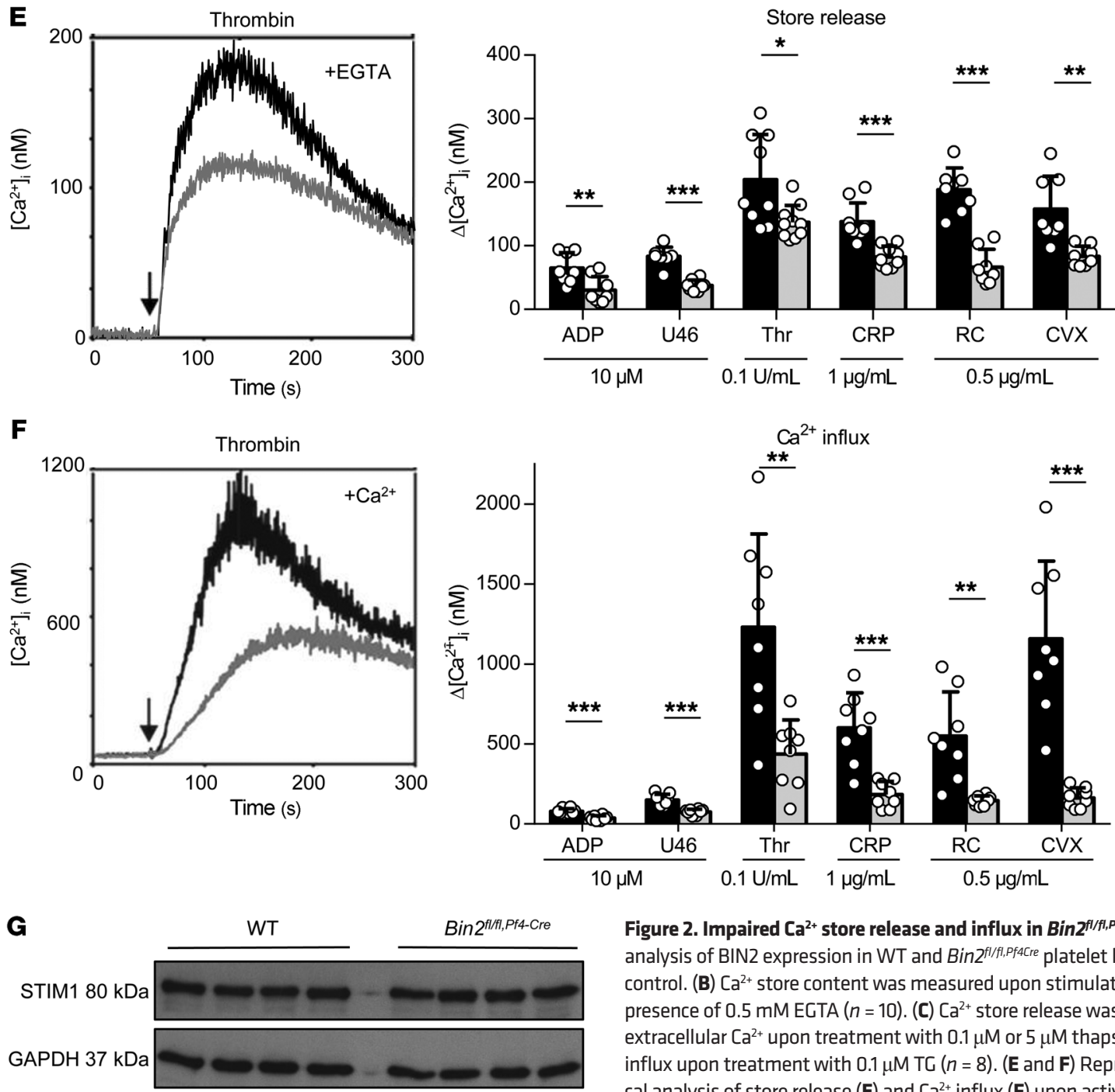

Figure 2. Impaired $\mathrm{Ca}^{2+}$ store release and influx in Bin $2^{f / f f, p f 4 c r e}$ platelets. (A) Western blot analysis of BIN2 expression in WT and Bin $2^{f / f f l, P f 4 c r e}$ platelet lysates with GAPDH as loading control. (B) $\mathrm{Ca}^{2+}$ store content was measured upon stimulation with $5 \mu \mathrm{M}$ ionomycin in the presence of 0.5 mM EGTA $(n=10)$. (C) $\mathrm{Ca}^{2+}$ store release was measured in the absence of extracellular $\mathrm{Ca}^{2+}$ upon treatment with $0.1 \mu \mathrm{M}$ or $5 \mu \mathrm{M}$ thapsigargin (TC) $(n \geq 8)$ and (D) $\mathrm{Ca}^{2+}$ influx upon treatment with $0.1 \mu \mathrm{M} \mathrm{TG}(n=8)$. ( $(\mathbf{E}$ and $\mathbf{F})$ Representative traces and statistical analysis of store release $(\mathbf{E})$ and $\mathrm{Ca}^{2+}$ influx $(\mathbf{F})$ upon activation of the platelets with the indicated agonists $(n \geq 8)$. (G) Western blot analysis of WT and Bin $2^{f / f f f, p f 4 C \text { re }}$ platelet lysates detecting the expression of STIM1 with GAPDH as loading control. Values are depicted as mean $\pm \mathrm{SD}$, and $P$ values were calculated using the Mann-Whitney $U$ test. ${ }^{*} P<0.05$, ${ }^{* *} P<0.01,{ }^{* *} P<0.001$. See complete unedited blots in the supplemental material.

restricted expression of BIN2, with the highest expression level detected in platelets (Figure 1A). To confirm the interaction of BIN2 and STIM1, we performed an IP with an anti-BIN2 antibody in human platelet lysate. We could demonstrate the presence of STIM1 in the pulldown after BIN2 precipitation by Western blot analysis (Figure 1B) and silver staining of the SDS-PAGE (Supplemental Figure 1C). Furthermore, we incubated the glutathione S-transferase-tagged (GST-tagged) STIM1 C-tail with recombinantly expressed BIN2 protein and performed a pulldown for GST. Using SDS-PAGE and silver staining, we detected the GST- 
tagged STIM1 $(\sim 50 \mathrm{kDa})$ and BIN2 $(\sim 70 \mathrm{kDa})$, demonstrating a direct interaction between the 2 proteins (Figure $1 \mathrm{C}$ ). The rather small amount of BIN2 precipitated in this experiment indicated a weak interaction of the 2 proteins. This was also reflected by a dissociation constant $\left(\mathrm{K}_{\mathrm{d}}\right)$ of approximately $196 \mu \mathrm{M}$, as determined in a fluorescence polarization assay, where increasing amounts of BIN2 were titrated against labeled STIM1. The shallow curve with a hill slope of less than 1 indicated a more complex binding mode, such as negative cooperativity, rather than a simple 1-site binding (Supplemental Figure 2).

BIN2-deficient mice are viable and fertile. In accordance with the expression pattern in humans, BIN2 expression in mouse tissue was also largely restricted to hematopoietic cells with the highest levels detectable in platelets (Figure 1D). The interaction between STIM1 and BIN2 was confirmed in mouse platelet lysates by IP of BIN2 and subsequent detection of STIM1 (Supplemental Figure 1, D and E).

To assess the function of BIN2 in vivo, we generated a con-

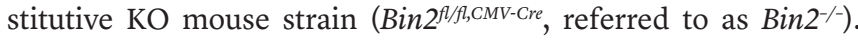
Homozygous Bin $2^{-/-}$mice were born at a normal Mendelian ratio, viable, and fertile and appeared healthy, indicating that BIN2, in contrast to STIM1 $(9,32)$ or Orai1 $(10,33)$, is dispensable for embryonic development. Western blot analysis confirmed the complete loss of BIN2 expression in all tested tissues (Figure 1D). Basic blood parameters, including platelet count and size, were not altered in Bin $2^{-/-}$mice compared with WT littermates (Supplemental Table 2).

To circumvent potential indirect effects of global BIN2 deficiency on platelet function, we used Pf4-Cre transgenic mice to generate a platelet/megakaryocyte-specific BIN2-KO mouse line $\left(B i n 2^{f l / f l, P f 4 C r e}\right)$. The absence of BIN2 protein in platelets of $\operatorname{Bin} 2^{f / f f, P f 4-C r e}$ mice was confirmed by Western blotting (Figure $2 \mathrm{~A}$ ). The basic blood parameters including platelet count, size, ultrastructure, and lifespan were unaltered compared with the respective littermate control (Supplemental Table 3 and Supplemental Figure 3, A and B). Investigations of cryosections of the bone marrow of $\operatorname{Bin} 2^{f l f f, P f 4-C r e}$ mice revealed no difference in megakaryocyte morphology and number (Supplemental Figure 3C). Of note, expression levels of glycoprotein V (GPV), GPVI, integrin $\beta 1$, and CD9 were slightly reduced in Bin2 $2^{f l f l, P f 4-C r e}$ platelets compared with the WT control (Supplemental Figure 3D).

Diminished $\mathrm{Ca}^{2+}$ responses in Bin $2^{f / f f, P f 4-C r e}$ platelets. Given the interaction between STIM1 and BIN2, we investigated the role of $\mathrm{BIN} 2$ in platelet $\mathrm{Ca}^{2+}$ homeostasis. We found comparable $\mathrm{Ca}^{2+}$ store content in Bin $2^{f / f f, P f 4-\mathrm{Cre}}$ and Bin $2^{f / / f l}$ littermate (WT) platelets, as measured by treatment of the cells with ionomycin in the absence of extracellular $\mathrm{Ca}^{2+}$ (WT: $344.2 \pm 104 \mathrm{nM}$; Bin2 ${ }^{f / f, f f 4-\mathrm{Cre}}: 316.2 \pm 55.25$ $\mathrm{nM}$ ) (Figure 2B and Supplemental Figure 4). Also, the $\mathrm{Ca}^{2+}$ store release evoked by the sarcoplasmic/ER $\mathrm{Ca}^{2+}$ ATPase (SERCA) pump inhibitor thapsigargin (TG) was indistinguishable between the 2 groups (WT: $73.75 \pm 14.89 \mathrm{nM}$; Bin2 $2^{f / f l, P f 4-C r e}: 83.78 \pm 15.48 \mathrm{nM}$ ) (Figure $2 \mathrm{C}$ and Supplemental Figure 4$)$. This is in line with the normal $\mathrm{Ca}^{2+}$ store content in Orai1-deficient platelets (10), but in contrast to STIM1 deficiency, where a reduced $\mathrm{Ca}^{2+}$ store content in platelets was observed (9), which points toward a role of STIM1 in $\mathrm{Ca}^{2+}$ store filling independent of BIN2. Remarkably, however, the TG-evoked $\mathrm{Ca}^{2+}$ influx in the presence of extracellular $\mathrm{Ca}^{2+}$ was diminished in the absence of BIN2 (WT: $877.5 \pm 391.2 \mathrm{nM}$; Bin2 $2^{f / f f, P f 4-\text { Cre }}: 264.6$ $\pm 143.6 \mathrm{nM}$ ) (Figure 2D and Supplemental Figure 4), suggesting a defect in the STIM1-dependent opening of Orai1 channels. Next, we studied receptor-dependent $\mathrm{Ca}^{2+}$ responses to GPCR agonists (ADP, thrombin, and the stable $\mathrm{TxA}_{2}$ analog U46619) and (hem) ITAM-dependent agonists acting on GPVI (collagen-related peptide, CRP and convulxin, CVX) or CLEC-2 (rhodocytin, RC). Interestingly, $\mathrm{Ca}^{2+}$ store release was significantly reduced in response to all tested agonists (Figure 2E and Supplemental Figure 5). In line with these findings and the results from the TG experiments, SOCE was also diminished in the mutant platelets, resulting in an overall severely defective $\mathrm{Ca}^{2+}$ response (Figure $2 \mathrm{~F}$ and Supplemental Figure 5). Together, these results clearly demonstrated a critical role of BIN2 in STIM1/Orai1-mediated SOCE, but also in agonist-induced $\mathrm{Ca}^{2+}$ store release. Of note, STIM1 expression levels were unaltered in Bin2 $2^{f / f t, P f 4-C r e}$ platelets (Figure $2 \mathrm{G}$ ).

Defective IP ${ }_{3} R$ function in Bin $2^{f / f f, P f 4-C r e}$ platelets. In order to identify the mechanisms underlying the defective agonist-induced $\mathrm{Ca}^{2+}$ store release, we analyzed the signal transduction pathways downstream of the (hem)ITAM receptors GPVI and CLEC-2. The phosphorylation of the key signaling molecules Syk (Y519/520), LAT (Y191), and PLC 2 (Y753) were unaltered in response to the GPVI agonist CVX or the CLEC-2 agonist RC (Figure 3, A and B). A central downstream pathway of all agonists is the PLC-dependent hydrolysis of $\mathrm{PIP}_{2}$ into DAG and $\mathrm{IP}_{3}$. In line with the unaltered (hem)ITAM tyrosine phosphorylation cascade, the amount of $\mathrm{IP}_{3}$ produced by Bin $2^{f / f, P f f-C r e}$ platelets upon activation with CVX or $\mathrm{RC}$ was indistinguishable from WT controls (Figure 3C). This was also the case for the GPCR agonist thrombin, suggesting that the defect was located downstream of $\mathrm{IP}_{3}$ generation. We therefore analyzed $\mathrm{IP}_{3} \mathrm{R}$ expression levels in the mutant platelets, but found them to be unaltered compared with WT controls (Figure 3D). To assess the functionality of $\mathrm{IP}_{3} \mathrm{R}$, we loaded platelets with inactive caged $\mathrm{IP}_{3}$, which can be activated with a UV light pulse during the measurement (34). Strikingly, in these experiments, both $\mathrm{Ca}^{2+}$ store release and SOCE were significantly reduced in Bin $2^{f / f f l, P f 4-\mathrm{Cre}}$ platelets compared with WT controls (Figure 3, E and F, and Supplemental Figure 5), strongly suggesting a functional defect of the $\mathrm{IP}_{3} \mathrm{Rs}$ in mutant platelets.

$B I N 2$ interacts with STIM1 and $I P_{3} R$. The results above indicated that BIN2 may not only interact with STIM1, but possibly also with $\mathrm{IP}_{3} \mathrm{Rs}$ to regulate $\mathrm{Ca}^{2+}$ signaling in platelets. To test this hypothesis directly, we performed a pulldown assay in which we incubated Bin $2^{f / f l f, P f 4-C r e}$ platelet lysates with recombinant Histagged BIN2. After purification with Ni-NTA beads, the different fractions were eluted and analyzed by immunoblotting. A high amount of BIN2 was detected in the eluate fractions (Figure $3 G$ ), demonstrating the functionality of the pulldown. Furthermore, STIM1 was robustly detected in the eluate fractions (Figure 3G), confirming the results obtained with human platelets (Figure 1B). In addition, we also identified $\mathrm{IP}_{3} \mathrm{R}$ in the eluate fraction of the pulldown, demonstrating a robust direct or indirect interaction of BIN2 with both STIM1 and $\mathrm{IP}_{3} \mathrm{R}$ in platelets. To ensure specificity, we screened for the unrelated protein RhoA, which was absent in the eluate fractions (Supplemental Figure 6A), and performed silver staining of the SDS-PAGE to visualize total protein content (Supplemental Figure 6B). 
A

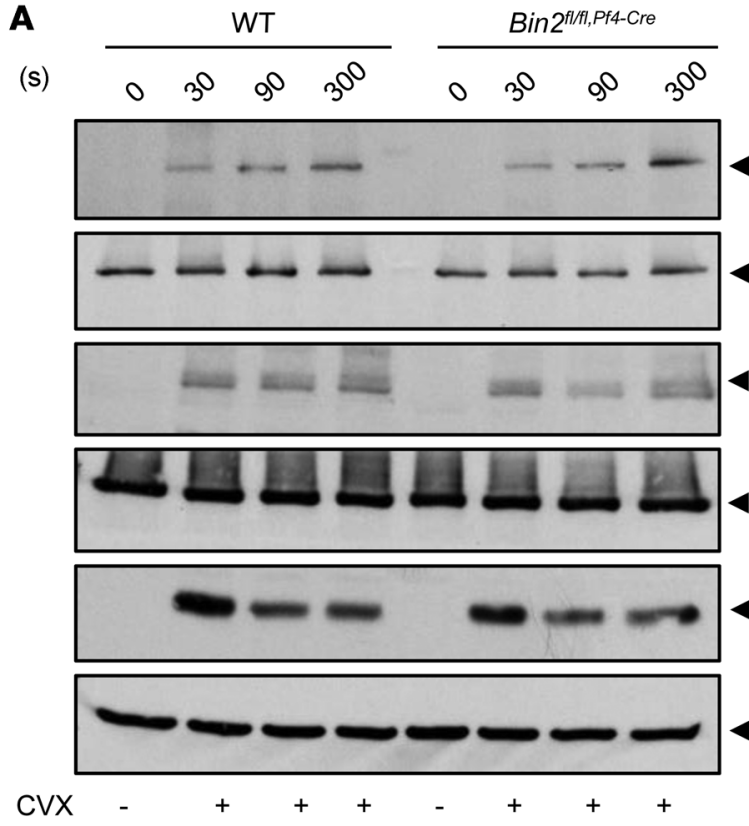

C

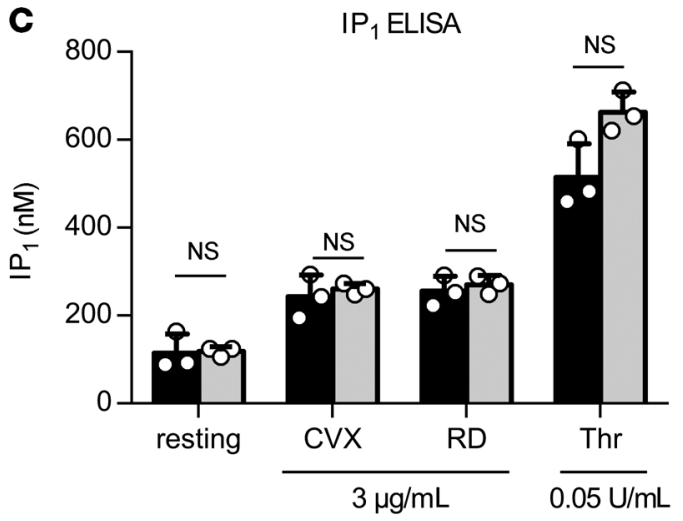

$\mathbf{F}$

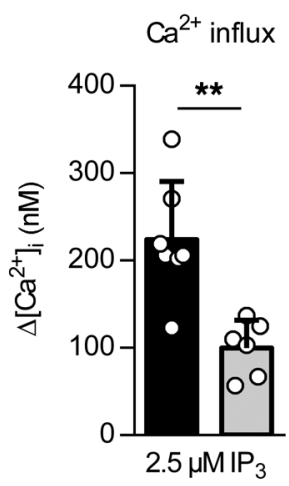

G

D

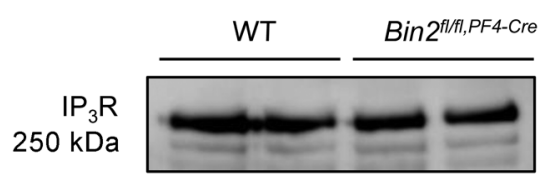

GAPDH $37 \mathrm{kDa}$

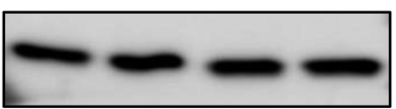

Bin2 $2^{\text {flffl, Pf4-Cre }}$

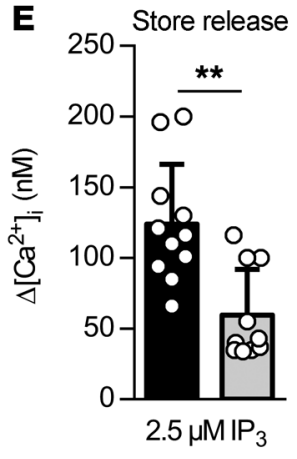

$2.5 \mu \mathrm{MIP}_{3}$
B

WT - $3^{\circ} \quad 9^{\circ} \quad 3^{\circ} \quad 3^{\circ} \quad 9^{\circ} \quad 3^{\circ}$ p-PLCY2 (Y759)

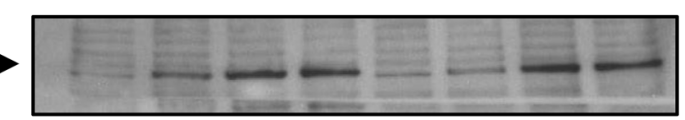

p-SYK $(Y 519 / 520)$
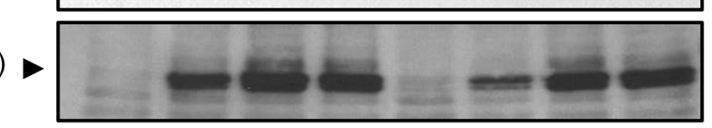

SYK

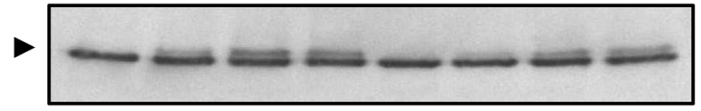

p-LAT (Y191)

LAT

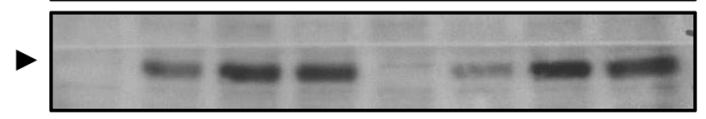
RC

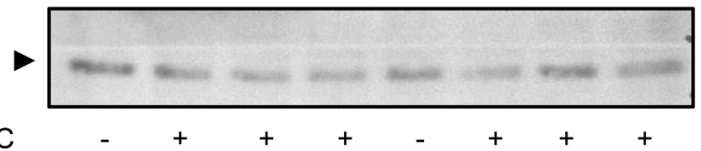

WT

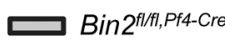

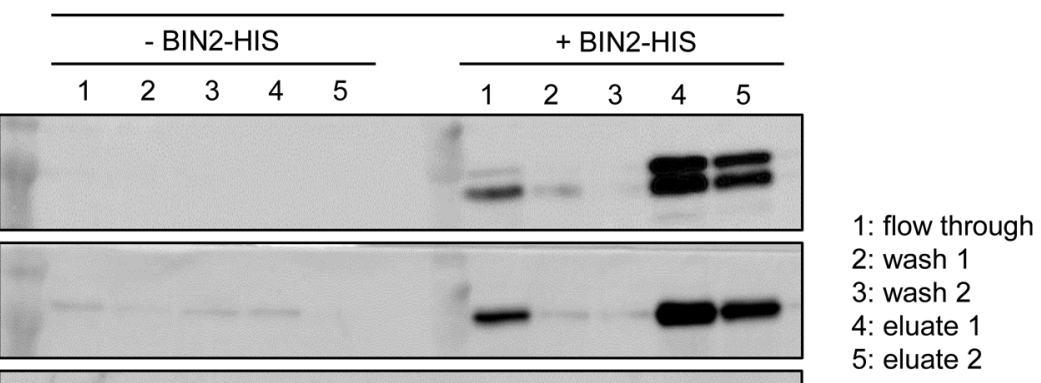

Figure 3. Defective $I P_{3} R$ function in Bin2 $2^{f / f, \text { Pfacre }}$ mice and BIN2 interaction with STIM1 and IP $\mathbf{R}$. (A and B) Determination of whole-cell tyrosine phosphorylation pattern in WT and Bin2 $2^{f l f f l, P f 4 c r e}$ platelets upon activation with convulxin (CVX) (A) or rhodocytin (RC) (B). The samples were taken at the indicated time points, lysed, and Western blot analysis was performed with the indicated phospho-specific and pan-antibodies. This blot is representative of 3 independent experiments. (C) Quantification of inositol monophosphate (IP), a specific metabolite of inositol-1,4,5-trisphosphate $\left(\mathrm{IP}_{3}\right)$, produced upon activation with the indicated agonist $(n=3)$. Representative results of 3 independent experiments. (D) Western blot analysis of IP $P_{3} R$ expression in WT and Bin2 $2^{f / f f, P f a c r e}$ platelet lysates; GAPDH was used as loading control. (E and F) Ca $\mathrm{a}^{2+}$ concentrations in the cytoplasm of WT and Bin2 $2^{f / f f l, P f 4 C r e}$ platelets upon treatment with UV light-inducible $\mathrm{IP}_{3}$ in the $(\mathbf{E})$ absence or $(\mathbf{F})$ presence of extracellular $\mathrm{Ca}^{2+}(n=8)$. (G) Binz $2^{f / f f l, \text { PfAcre }}$ platelet lysates were incubated with recombinant BIN2-HIS protein, followed by a purification step with NI-NTA beads. The different fractions were eluted and analyzed by Western blotting using BIN2-, STIM1-, and IP ${ }_{3}$-specific antibodies. Representative result of 3 independent experiments. Values are depicted as mean \pm SD, and $P$ values were calculated using the Mann-Whitney $U$ test. ${ }^{* *} P<0.01$. See complete unedited blots in the supplemental material. 
A
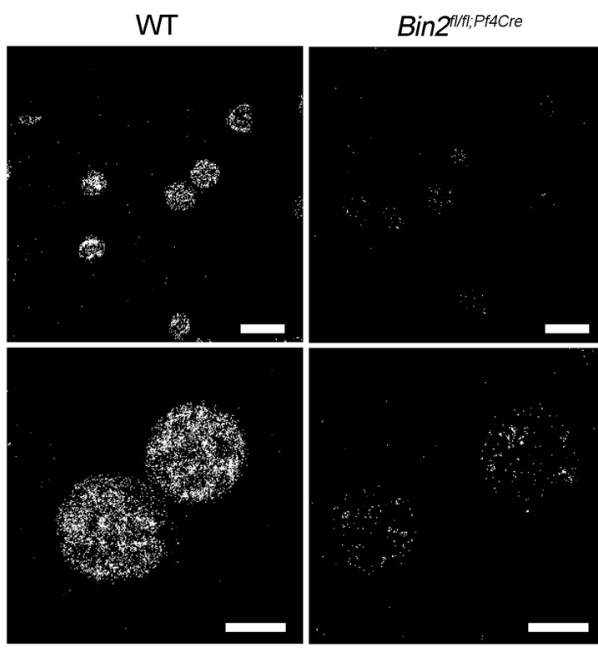

C

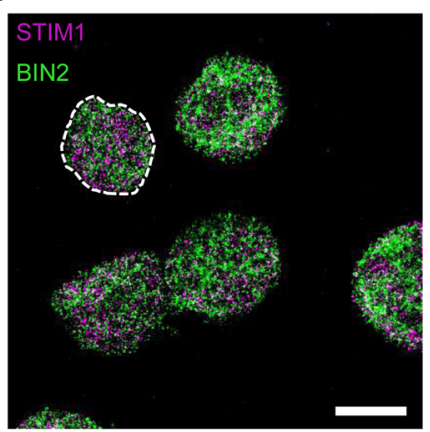

D
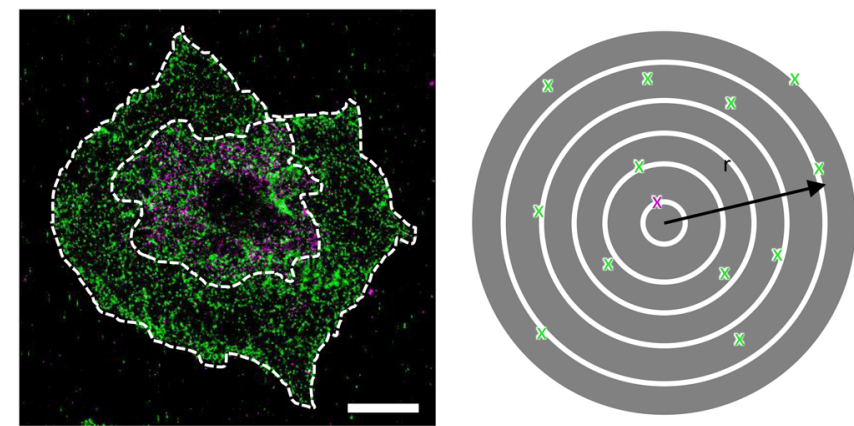

$\mathbf{F}$

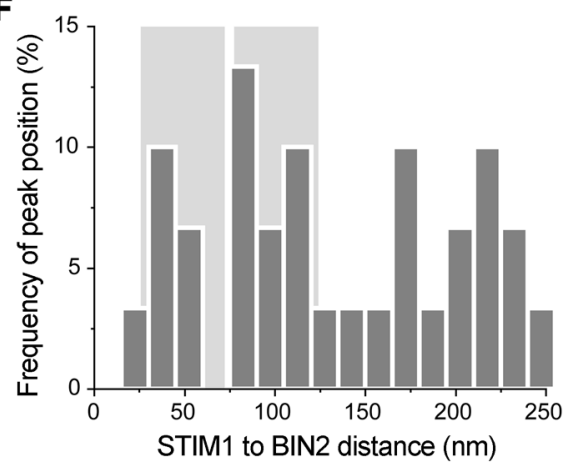

E

B
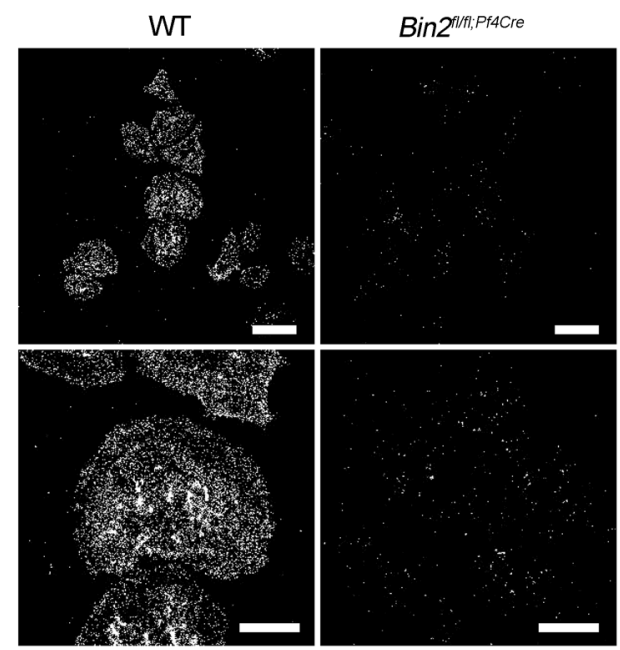

G

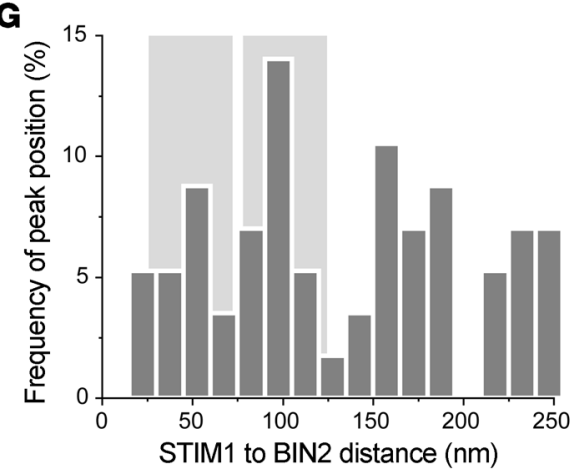

Figure 4. BIN2 STIM1 colocalization in human platelets. dSTORM images of (A) resting mouse platelets on glycine or (B) thrombin-activated WT and Bin2 $2^{f / f f, P f 4 C r e}$ mouse platelets on fibrinogen, stained for BIN2. Scale bars $5 \mu \mathrm{m}$ (upper panel) and $2 \mu \mathrm{m}$ (lower panel). Dualcolor dSTORM allows resolution of the distribution of STIM1 (magenta) and BIN2 (green) in (C) resting and (D) activated human platelets; dashed white lines indicate areas of neighbor density analysis (E) between STIM1 (magenta) and BIN2 (green), with discrete ring areas in which the BIN2 density was determined (dark gray). In activated spread platelets, only localizations in the inner region where the organelles and intercellular membrane compartments accumulate were considered for STIM1. Colocalization hotspots of STIM1 and BIN2 are identified by analyzing the BIN2 density at increasing radial distances to STIM1: Histograms of the distance-dependent BIN2 density peak maxima found for (F) resting ( $n=6$, Supplemental Figure 7A) and (G) activated human platelets ( $n=14$, Supplemental Figure 7B). The distinct STIM1 to BIN2 distance regions with increased accumulation of colocalization hotspots are highlighted in light gray. Scale bars (C and D): $2 \mu \mathrm{m}$.
To investigate the localization of BIN2 in murine platelets, we used super-resolution microscopy by direct stochastic optical reconstruction microscopy $(d \mathrm{STORM})(35,36)$ in combination with a newly in-house-generated monoclonal anti-mouse BIN2 antibody. Resting platelets were immobilized on glycine, whereas thrombin-activated platelets were allowed to spread on fibrinogen before the staining procedure. An inhomogeneous distribution of BIN2 in the cytoplasm was detected in resting WT platelets, whereas no staining was observed in Bin $2^{f / f l \text {, Pf4-Cre }}$ platelets (Figure 4A). In spread WT platelets, BIN2 was mainly located in the cell center, with multiple sites of clustering (Figure 4B). These results indicated accumulation of BIN2 at internal membranes.

In human platelets, dual-color $d$ STORM imaging allowed us to visualize the distribution of BIN2 and STIM1 in resting platelets on glycine- and thrombin-activated spread platelets on fibrinogen (Figure 4, C and D). A neighbor density analysis of the distance-dependent BIN2 density around STIM1 localization events confirmed the accumulation of BIN2 in close proximity to STIM1 (Figure 4, E-G, and Supplemental Figure 7). Most notable, an increased BIN2 density was consistently found at distinct distances of approximately 50 and $100 \mathrm{~nm}$ (see light gray highlighted areas in Figure 4, F and G) for both activated and resting platelets. These findings strongly suggest colocalization hotspots of STIM1 and BIN2 in human platelets. The exact molecular constellation of the STIM1/BIN2 interaction, however, could not be resolved because of the large size of IgG antibodies of approximately 10 to $15 \mathrm{~nm}$ (37). Note that comparative colocalization studies in mouse platelets were not possible because of the lack of functional $d$ STORM antibodies for mouse STIM1. 
A

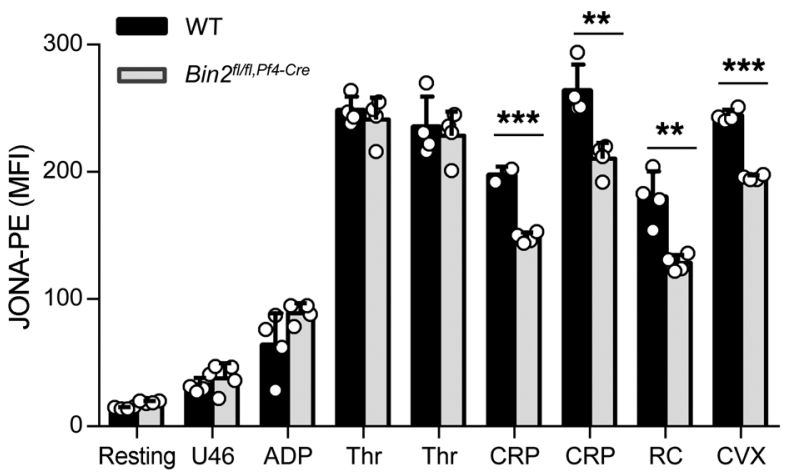

B

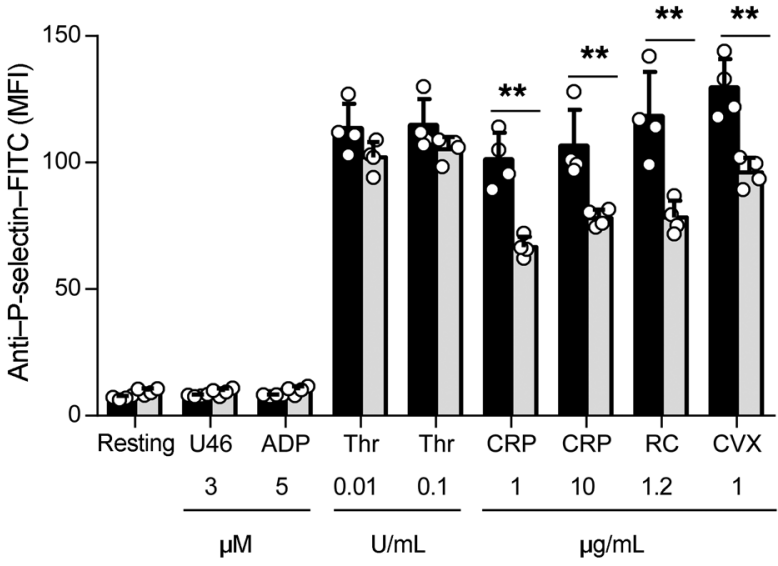

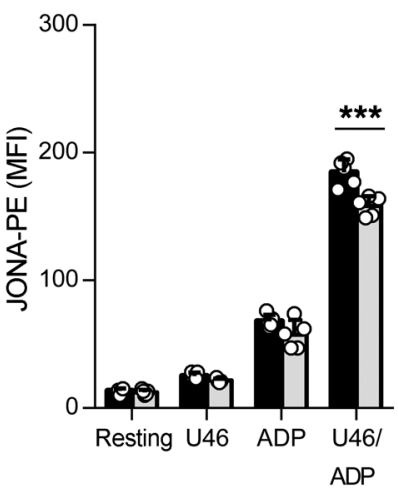

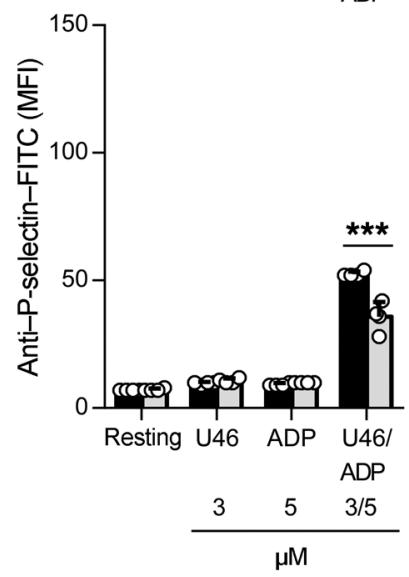

D

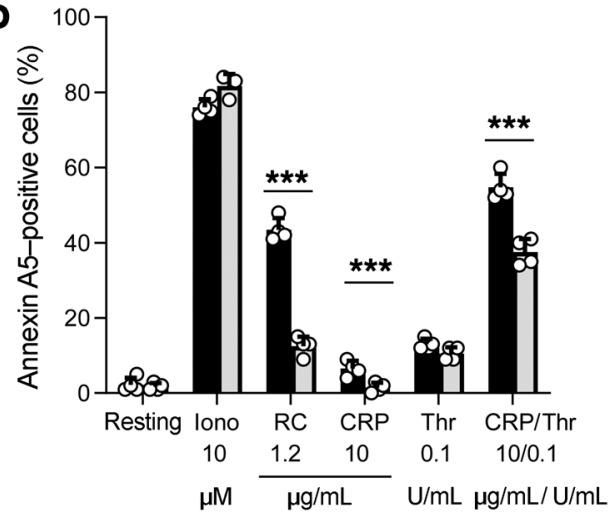

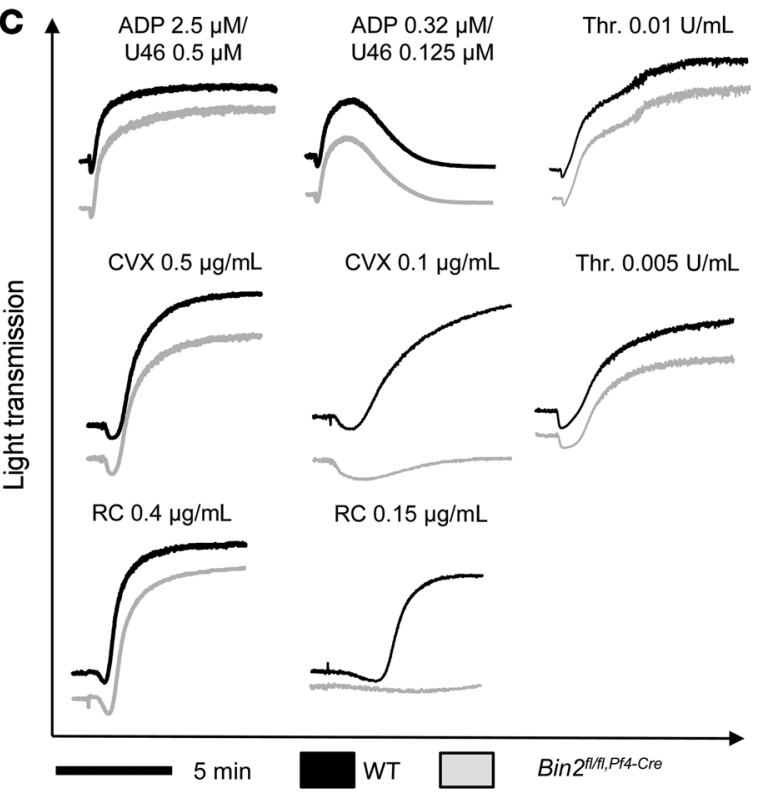

Figure 5. Defective integrin activation and aggregation of Bin2 $2^{f / f f, p f 4 c r e}$ platelets upon (hem)ITAM stimulation. (A and $\mathbf{B}$ ) Flow cytometric analysis of $(\mathbf{A})$ integrin $\alpha$ llb $\beta 3$ activation (binding of JON/A-PE) and (B) degranulation-dependent P-selectin exposure in WT and Bin ffl/ff,pfacre platelets in response to the indicated agonists ( $n=$ 4). Representative result of 3 independent experiments. Values are depicted as mean $\pm \mathrm{SD}$, and $P$ values were calculated using the Mann-Whitney $U$ test. ${ }^{* *} P<0.01$, ${ }^{* * *} P$ $<0.001$. (C) Washed platelets were stimulated with the indicated agonists, and light transmission was recorded using a 4-channel aggregometer. Representative results of 3 independent experiments. (D) Phosphatidylserine exposure of platelets in response to the indicated agonists was measured by flow cytometry. $n \geq 5$. Representative result of 3 independent experiments. Values are depicted as mean \pm SD, and $P$ values were calculated using the Mann-Whitney $U$ test. ${ }^{* *} P<0.01$, ${ }^{* * *} P<0.001$.

Defective activation responses in Bin $2^{f / f, P f-C r e}$ platelets. The above

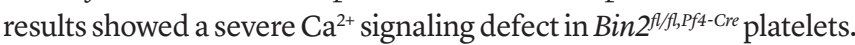
To evaluate the functional consequences of the observed $\mathrm{Ca}^{2+}$ defect on the activation of Bin $2^{f / f, P f f-C r e}$ platelets, we performed flow cytometric analysis of agonist-induced integrin $\alpha \operatorname{IIb} \beta 3$ activation and
P-selectin surface exposure, a marker of $\alpha$-granule release (Figure

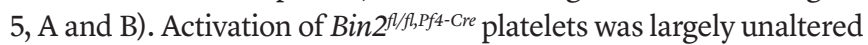
in response to the GPCR agonists ADP, thrombin, and U46619, with the exception of a weak but significant reduction upon costimulation with U46619 and ADP. However, the response of Bin $2^{A / A, P f f-C r e}$ plate- 
A

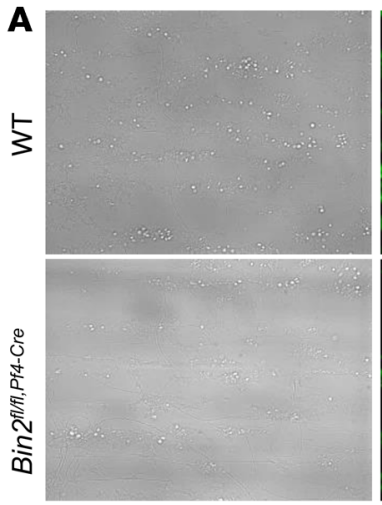

B

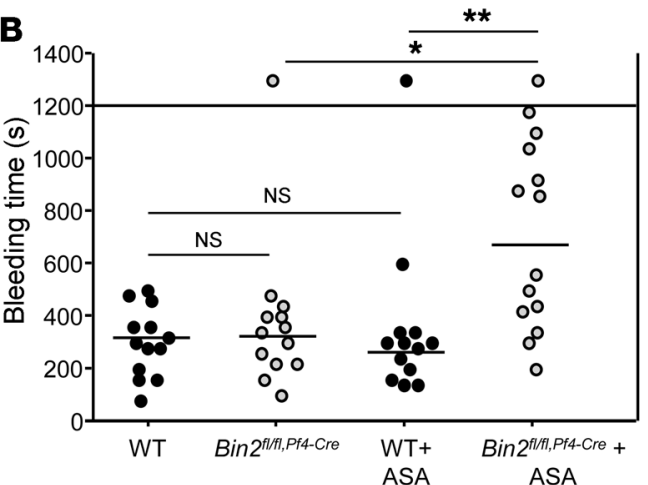

E

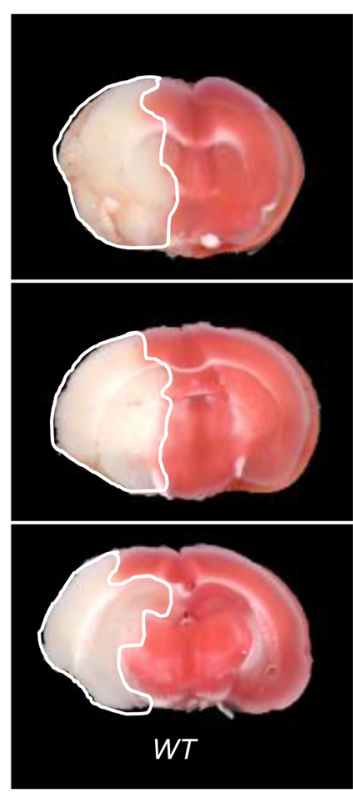

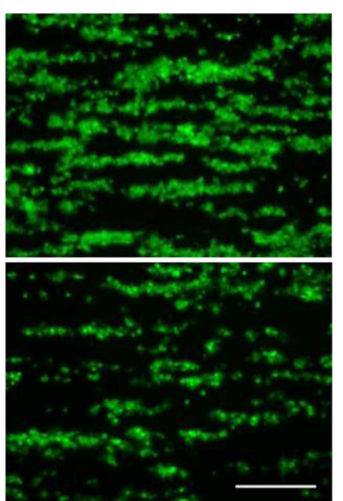

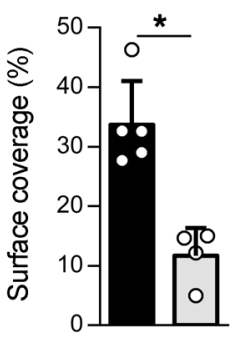

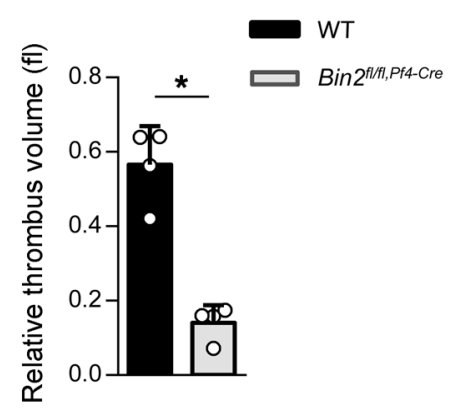

C

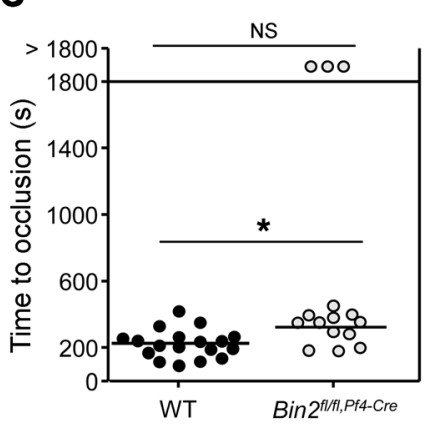

$\mathbf{F}$

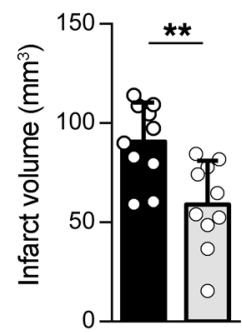

H

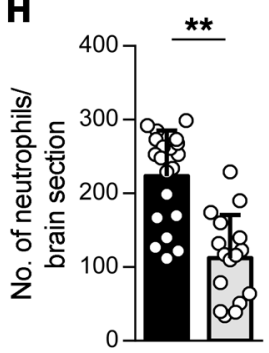

G
D
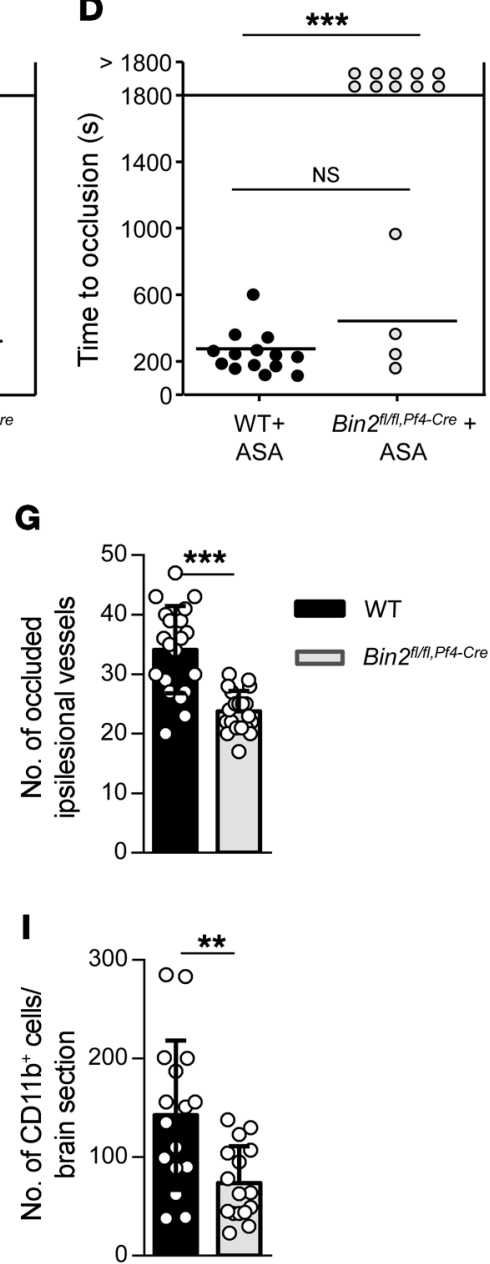

Figure 6. Impaired hemostasis, defective arterial thrombus formation, and protection from ischemic brain infarction in Bin2 ${ }^{f / f f l, P f 4 c r e}$ mice. (A) Adhesion and thrombus formation of platelets on collagen was assessed in a flow adhesion assay at a wall shear rate of 1700/s. Representative images and the quantification of the surface coverage and the relative thrombus volume are shown $(n=4)$. Representative example of 3 independent experiments. Values are depicted as mean \pm SD, and $P$ values were calculated using the Mann-Whitney $U$ test. ${ }^{*} P<0.05$. Scale bar: $50 \mu \mathrm{m}$. (B) Tail bleeding times of WT and

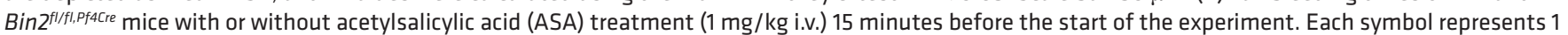
animal. Kruskal-Wallis test ( $P$ value: 0.0022 ) followed by Dunn's multiple-comparisons post hoc test; ${ }^{*} P<0.05,{ }^{* *} P<0.01$. (C and D) Time to stable vessel occlusion of WT and Bin $2^{f / f f l, P f 4 c r e}$ mice without (C) or with (D) ASA treatment (1 mg/kg i.v.) 15 minutes before the start of the experiment. The abdominal aorta was injured by firm compression with forceps, and blood flow was monitored for 30 minutes. Mann-Whitney $U$ test was performed to compare mean occlusion times of occluded vessels. To compare frequency of occluded and nonoccluded vessels, Fisher's exact test was used. ${ }^{*} P<0.05,{ }^{* * *} P<0.001$.

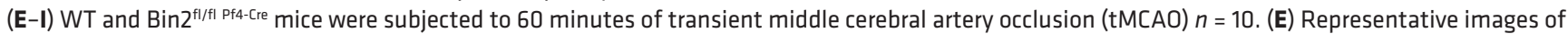
3 coronal brain sections stained with TTC. (F) Infarct volumes, (C) number of occluded ipsilateral vessels (2 slides per animal analyzed), (H) number of neutrophils, and (I) CD11 b+ cells per section (2 slides per animal analyzed) were investigated after 24 hours. Values are depicted as mean \pm SD, and $P$ values were calculated using the Mann-Whitney $U$ test. ${ }^{* *} P<0.01,{ }^{* * *} P<0.001$. 
lets toward the GPVI agonists CRP or CVX was significantly reduced compared with WT controls. Similar observations were made upon stimulation with the CLEC-2 agonist RC (Figure 5, A and B). In line with the decreased integrin activation, aggregation responses to (hem)ITAM-specific stimulation were reduced, which was most evident at low agonist concentrations (Figure 5C). No differences in aggregation upon stimulation with the GPCR agonists thrombin, ADP, and U46619 or the combination of ADP/U46619 were detected (Figure $5 \mathrm{C}$ and Supplemental Figure $6 \mathrm{C}$ ). These results are in accordance with previous studies showing that the $\mathrm{Ca}^{2+}$ defects observed in STIM1- and Orai1-deficient platelets are of particular functional relevance for (hem)ITAM-mediated, but to a much lesser extent GPCR-mediated, activation responses under static conditions (8-10). Since $\mathrm{Ca}^{2+}$ and SOCE are of particular importance for phosphatidylserine (PS) exposure upon platelet activation, we investigated this process by measuring the ability of Bin $2^{f / f f, P f 4-C r e}$ platelets to bind annexin A5 in flow cytometry. We found significantly reduced PS exposure in response to the tested agonists in the mutant platelets compared with the WT control (Figure 5D). Of note, Bin $2^{f / f f, P f 4-\mathrm{Cre}}$ platelets showed unaltered spreading on fibrinogen, indicating that BIN2 is neither required for integrin $\alpha \operatorname{IIb} \beta 3$ outside-in signaling nor for the rapid rearrangements of the actin cytoskeleton that underlie this process (Supplemental Figure 6D and ref. 38). This is in line with normal shape change observed in aggregometry (Figure 5C) and unaltered ultrastructure (Supplemental Figure 3B).

Thrombus formation at sites of vascular injury requires stable shear-resistant platelet adhesion on the extracellular matrix and auto- and paracrine platelet activation by locally released secondary mediators. When whole blood was perfused over immobilized collagen at a shear rate of $1700 / s$, WT platelets adhered rapidly to the collagen-coated surface and recruited additional platelets, resulting in the formation of stable 3-dimensional aggregates. In sharp contrast, adhesion and aggregate formation of $\operatorname{Bin} 2^{f l / f, P f 4-\mathrm{Cre}}$ platelets was severely impaired, resulting in a $65 \%$ and $75 \%$ reduction in surface coverage and thrombus volume, respectively (Figure 6A). This is in line with the observations made with STIM1- and Orai1-deficient platelets and underlines the importance of $\mathrm{Ca}^{2+}$ signaling in platelets under shear flow conditions (39). Furthermore, the reduced PS exposure of Bin $2^{f / f f, P f 4-C r e}$ platelets was also confirmed in this experimental setting (Supplemental Figure 6E).

In summary, these results demonstrated impaired integrin activation, degranulation, and aggregation of $\operatorname{Bin} 2^{f / f f l, P f 4-C r e}$ platelets in response to (hem)ITAM agonists under static conditions and defective shear-resistant aggregate formation under flow in vitro.

Impaired hemostasis, defective arterial thrombus formation, and reduced thrombo-inflammatory brain infarction in Bin $2^{f l f f l, P f 4-\mathrm{Cre}}$ mice. To examine the extent to which the observed defects in $\mathrm{Ca}^{2+}$ homeostasis of Bin $2^{f / f f, P f 4-C r e}$ platelets influenced hemostasis, we performed a tail-bleeding assay. Here, a $2 \mathrm{~mm}$ segment of the tail tip was cut off with a scalpel and the time until bleeding ceased was determined. No differences between $\operatorname{Bin} 2^{f / f f l, P f 4-C r e}$ and WT mice were observed (Figure 6B), confirming the previous finding that even severely impaired $\mathrm{Ca}^{2+}$ signaling in platelets per se does not cause a major hemostatic defect (8-10).

Next, we investigated occlusive thrombus formation in the abdominal aorta after mechanical injury of the vessel by compression with forceps. The time to vessel occlusion was significantly prolonged in Bin2 $2^{f / f t, P f 4-\mathrm{Cre}}$ mice compared with WT mice, and 3 out of 14 vessels did not occlude during the observation time of $30 \mathrm{~min}-$ utes (mean occlusion time: WT: $230 \pm 85$ seconds; Bin2 $2^{f / f f, P f 4-C r e}: 323$ \pm 90 seconds; $P=0.012$; Figure $6 \mathrm{C}$ ). It has been shown that defective GPVI signaling can be partially compensated for by $\mathrm{TxA}_{2}-\mathrm{me}-$ diated $G_{q} / G_{13}$-induced signaling pathways, which enables platelets to further deplete the $\mathrm{Ca}^{2+}$ stores and to enhance SOCE (40-42). Therefore, we investigated the role of $\mathrm{TxA}_{2}$-induced signaling in vivo by inhibiting TxA $A_{2}$ synthesis in WT and $\operatorname{Bin} 2^{f f f f, P f 4-C r e}$ mice by intravenous administration of a low dose of acetylsalicylic acid (ASA: $1 \mathrm{mg} / \mathrm{kg}$ bodyweight) and subsequently assessed tail bleeding times and occlusive thrombus formation in the injured aorta. ASA treatment had no major effect in WT mice, but resulted in significantly prolonged tail bleeding times in Bin2 $2^{f / f, P f 4 C r e}$ mice (Figure 6B). Similarly, ASA-treated WT mice developed occlusive thrombi in the mechanically injured aorta, whereas only 4 out of 14 vessels in ASA-treated Bin $2^{f / f f, P f 4-C r e}$ mice occluded $(P=0.0002$; Figure 6D). Together, these data confirmed that a functional defect in (hem) ITAM-dependent platelet activation can be partially compensated for by $\mathrm{TxA}_{2}$-induced signaling pathways under in vivo conditions.

Platelets are increasingly recognized as key modulators of inflammatory responses, and their concerted action with components of the immune system to cause tissue damage is referred to as thrombo-inflammation. This was first described in the setting of (experimental) stroke, where platelets and T cells drive secondary thrombo-inflammatory brain infarct growth despite successful recanalization of the previously occluded brain artery. Recent experimental data has highlighted a critical role of the platelet GPVI/LAT/PLC $\gamma 2 / S T I M 1 / O r a i 1$ axis in this process $(9,10,43)$, and increased expression levels of STIM1/Orai1 have been proposed to serve as a predictor for poor outcome of stroke (44). To assess the pathophysiological significance of BIN2-dependent SOCE in this process, we subjected $\operatorname{Bin} 2^{f / f f, P f 4-C r e}$ mice to the transient middle cerebral artery occlusion (tMCAO) model of ischemic stroke, in which the middle cerebral artery (MCA) was transiently occluded by insertion of a filament as described previously (45). After removal of the filament, the animals were monitored for another 24 hours before the infarct sizes were assessed quantitatively on 2,3,5-triphenyl tetrazolium chloride-stained (TTCstained) brain slices (Figure $6 \mathrm{E}$ ). In Bin $2^{f / f f, P f 4-C r e}$ mice, infarct volumes were reduced by more than $30 \%$ compared with control mice (WT: $90 \pm 6 \mathrm{~mm}^{3}$, Bin2 $2^{f / f f, P f 4-\mathrm{Cre}}: 59 \pm 7 \mathrm{~mm}^{3} ; P=0.003$; Figure $6 \mathrm{~F})$. The number of occluded vessels in the ipsilateral hemisphere, indicative of tissue damage and resultant thrombotic activity, as well as the number of accumulated neutrophils and CD11b cells, were reduced in the ipsilateral hemispheres of Bin2 $2^{f / f l, P f 4-C r e}$ mice compared with WT littermates (Figure 6, G-I). In summary, these results demonstrated that BIN2 deficiency in platelets results in defective thrombus formation under flow and provides profound protection against arterial thrombosis and amelioration of thrombo-inflammatory brain infarction.

\section{Discussion}

This study is, to our knowledge, the first to describe an adapter protein that regulates both $\mathrm{IP}_{3} \mathrm{R}$ and STIM1 function in platelets, thereby connecting $\mathrm{Ca}^{2+}$ store release and SOCE function. Our data demonstrated a direct interaction of BIN2 with STIM1; how- 
ever, the weak binding affinity and the more robust interaction of the proteins in cell lysates strongly suggests that this interaction is stabilized by additional binding partners in a multiprotein complex at the $\mathrm{Ca}^{2+}$ stores.

The first evidence that $\mathrm{IP}_{3} \mathrm{R}$ can modify $\mathrm{Ca}^{2+}$ influx not only indirectly by releasing $\mathrm{Ca}^{2+}$ from the internal store, but also by a direct effect on major regulators of $\mathrm{Ca}^{2+}$ entry, was its reported interaction with transient receptor potential channels in HEK cells (46). Conversely, another study revealed a regulating effect of STIM1 on the activity of $\mathrm{IP}_{3} \mathrm{R}$ by direct interaction of both proteins in bovine aortic endothelial cells (47). Also, in STIM1-KO, but not in ORAI1-KO platelets, a reduced store release was detected in response to all tested agonists $(9,10)-$ however, because of the diminished store content in these platelets it was difficult to make a direct conclusion about the functional influence of STIM1 on $\mathrm{IP}_{3} \mathrm{R}$. Furthermore, immobile $\mathrm{IP}_{3} \mathrm{R}$ clusters, responsible for the $\mathrm{Ca}^{2+}$ release from the ER, colocalize with STIM1 puncta that form upon activation of SOCE (16). The association of IP ${ }_{3}$ to STIM1 has been shown to provide a reduced calcium microenvironment near the STIM1 EF-hand, its calcium-sensing part residing inside the $\mathrm{Ca}^{2+}$ store, thereby facilitating puncta formation and enhancing Orai1 activation and SOCE (17). Based on the data presented here, it is tempting to speculate that BIN2 may be involved in the formation of these $\mathrm{IP}_{3} \mathrm{R}$-STIM1 complexes. Of note, $\mathrm{IP}_{3} \mathrm{R}$ was not detectable in IP experiments with either human or mouse platelet lysate (data not shown). This might be a consequence of the low amount of BIN2 protein precipitated in the IP experiments compared with the concentration of recombinant BIN2 in pulldown experiments. Alternatively, BIN2 might regulate $\mathrm{IP}_{3} \mathrm{R}$ function independently of direct or indirect physical interactions with the receptor, e.g., by modulating the complex upstream regulatory signaling pathways that control IP $\mathrm{I}_{3} \mathrm{R}$ function. These include small molecules, such as nucleotides, as well as protein kinases and phosphatases $(48,49)$. Further studies will be required to unravel the exact underlying molecular mechanisms.

Super-resolution microscopy data also support the BIN2STIM1 interaction. Particularly, our neighbor density analysis (50) revealed an accumulation of BIN2 in close proximity to STIM1 at characteristic distance regions of 50 and $100 \mathrm{~nm}$ (for both activated and resting human platelets). These findings strongly suggest colocalization hotspots of STIM1 and BIN2 that have not been described before to our knowledge. Such hotspots may form due to steric hindrance induced by the large size of IgG antibodies used in immunolabeling (37). It is likely that the $50 \mathrm{~nm}$ pattern represents the minimal possible distance, with the width of each peak mostly indicating the flexibility of the linkers.

Of note, Ambily et al. (51) previously performed IP of STIM1 protein from TG-treated human platelet lysates and identified 4 potential interaction partners, which did not, however, include BIN2. This rather low number of proteins identified might be a consequence of lower sensitivity by precipitating limited amounts of endogenous STIM1 protein with an antibody. Of the 4 molecules identified in their study, myosin- 9 and actin also yielded high scores in our experiments; however, we did not identify DOCK10 or thrombospondin. This might be explained by methodological differences, e.g., by the use of a truncated STIM1 construct in our study or the TG pretreatment of platelets in the Ambily et al. study.
Interestingly, BIN1, another member of the BIN protein family, has recently been identified as regulating the microtubulemediated transport and surface expression of the calcium channel Cav1.2. in cardiomyocytes (27). Cardiomyocyte-specific loss of BIN1 results in a decreased number of protective membrane folds of T-tubules and alters cation diffusion within the T-tubules, thus increasing the duration of action potentials (28). Of note, we found that the ultrastructure, as well as the ability to change the morphology during spreading, was not altered in BIN2-deficient platelets (Supplemental Figure 3B and Supplemental Figure 6D), indicating that BIN2 exerts its effect on $\mathrm{Ca}^{2+}$ signaling via a different mechanism. As previously described for mice lacking STIM1 (9) or Orai1 (10), the severe SOCE defect in Bin2 $2^{f / f, P f 4-C r e}$ platelets hardly affected their functional responses to GPCR agonists, but impaired cellular activation through (hem)ITAM signaling. The difference in SOCE dependence between these 2 major activation pathways is currently not understood, but may be related to different kinetics in $\mathrm{IP}_{3}$ production because the (hem)ITAM/PLC $\gamma 2$ axis responds more slowly to a stimulus compared with the GPCR/PLC $\beta$ signaling.

Of note, we also observed a slightly decreased expression of some surface glycoprotein receptors on Bin $2^{f / f l f, P f 4-C r e}$ platelets. Since these alterations were very minor, we consider it very unlikely that they significantly contributed to the observed platelet-related phenotype of Bin2-KO mice.

The viability of the Bin2 $2^{-/-}$animals, as well as the unaltered blood parameters, demonstrated that BIN2 is dispensable for embryonic development. This is in contrast to STIM1 and Orai1 mutant animals, which die perinatally $(8-10,32)$, indicating that these molecules may not be suitable pharmacological targets for long-term treatment of thrombotic diseases because of undesired effects on different tissues, including immune cells and skeletal muscle, which are also observed in patients with STIM1 or ORAI1 mutations (52). The more restricted expression of BIN2 in the hematopoietic system and its central role in $\mathrm{Ca}^{2+}$ signaling in platelets provides proof of principle that the adaptors involved in $\mathrm{Ca}^{2+}$ signaling vary between different cell types, and might thereby be good targets to selectively inhibit or modulate the $\mathrm{Ca}^{2+}$ signaling machinery in a cell type-selective manner. Remarkably, platelet-specific BIN2 deficiency significantly reduced cerebral infarct growth in a model of acute ischemic stroke without increasing the risk of intracranial hemorrhage. Although further studies will be required to assess the druggability of BIN2, our findings corroborate the concept that molecules modulating $\mathrm{Ca}^{2+}$ homeostasis specifically in platelets may become promising novel targets for the prevention and/or treatment of thrombotic and thrombo-inflammatory diseases with minimal effects on normal hemostasis.

\section{Methods}

\section{Animals}

Generation of Bin $2^{f / f l}$ mice. A $9.96 \mathrm{~kb}$ region used to construct the targeting vector was designed such that the $5^{\prime}$ homology arm extended about $2.83 \mathrm{~kb} \mathrm{5}$ ' to the $3^{\prime}$ end of the LoxP-FRT-flanked Neo cassette. The $3^{\prime}$ homology arm ended 3 ' from the single LoxP cassette and was $6.52 \mathrm{~kb}$ long. The LoxP-FRT-flanked Neo cassette was inserted 403 bp upstream of exon 3. The single LoxP site was inserted 157 bp down- 
stream of exon 3. DNA of the targeting vector was linearized by NotI digestion and then transfected by electroporation of BA1 (C57BL/6 $\times$ $129 /$ SvEv) (hybrid) embryonic stem cells. After selection with G418, surviving clones were expanded for PCR analysis; 5 positive clones were identified and expanded. Targeted iTL BA1 (C57BL/6N $\times 129 /$ SvEv) hybrid embryonic stem cells were microinjected into blastocysts of pseudopregnant C57BL/6 females. Highly chimeric offspring were mated to C57BL/6 FLP mice to remove the Neo cassette and backcrossed to $\mathrm{C} 57 \mathrm{BL} / 6$ females, resulting in $\mathrm{Bin} 2^{f l /+}$ mice. Constitutive $\mathrm{KO}$ mice and megakaryocyte/platelet-specific $\mathrm{KO}$ mice were generated by mating with CMV-Cre or PF4-Cre mice, respectively $(53,54)$. Intercrossing of the respective offspring resulted in Bin $2^{f / f l, C M V-C r e t /-}$ (referred to as Bin2 $2^{-/-}$) mice or Bin $2^{f / f f, P f f-C r e+/-}$ mice. Bin $2^{f / f f, P f 4-C r e-/-}$ littermates served as controls.

\section{Transformation of competent $E$. coli cells}

For transformation, chemically competent E. coli BL21 (DE3) or E. coli BL21 (DE3) pRARE2 cells were incubated with 50 ng plasmid DNA of Stim1 C-tail (aa 484-685; Supplemental Figure 1A) in pGEX6p1 or Bin2 in pETM11, respectively, for 30 minutes on ice. Transformation was triggered by a 45 -second heat shock at $42^{\circ} \mathrm{C}$ with subsequent incubation for 3 minutes on ice. Thereafter, bacteria were supplied with $500 \mu \mathrm{L} \mathrm{LB}$ medium and incubated at $450 \mathrm{rpm}$ for 1.5 hours at $37^{\circ} \mathrm{C}$. Positive clones were selected by plating the transformation mixture on LB-agar plates $(10 \mathrm{~g} / \mathrm{L}$ Bacto tryptone, $5 \mathrm{~g} / \mathrm{L}$ Bacto yeast extract, $5 \mathrm{~g} / \mathrm{L} \mathrm{NaCl})$ containing the respective antibiotics $(50 \mu \mathrm{g} / \mathrm{mL}$ Amp and $34 \mu \mathrm{g} / \mathrm{mL}$ Cam for Stim1 C-tail in pGEX6p1 or $50 \mu \mathrm{g} / \mathrm{mL}$ Kan for Bin2 in pETM11 and $34 \mu \mathrm{g} / \mathrm{mL} \mathrm{Cam}$ ) and incubated overnight at $37^{\circ} \mathrm{C}$.

\section{Protein production}

A single colony was used to inoculate $25 \mathrm{~mL} \mathrm{LB}$ (for Stim1 C-tail in pGEX6p1) or $100 \mathrm{~mL}$ terrific broth (TB) (12 g/L tryptone, $24 \mathrm{~g} / \mathrm{L}$ yeast extract, $4 \mathrm{~mL} / \mathrm{L}$ glycerol, $0.017 \mathrm{M} \mathrm{KH}_{2} \mathrm{PO}_{4}, 0.072 \mathrm{M} \mathrm{K}_{2} \mathrm{HPO}_{4}$ ) medium (for Bin2 in pETM11) containing antibiotics at $37^{\circ} \mathrm{C}$ and 200 rpm overnight. The next day, these precultures were used to inoculate $2.5 \mathrm{~L} \mathrm{LB}$ or $2 \mathrm{~L} \mathrm{~TB}$ medium supplemented with antibiotics and cultivated at $37^{\circ} \mathrm{C}$ and 200 rpm until bacterial concentration reached an $\mathrm{OD}_{600}$ of 0.4-0.6. Protein expression was induced by addition of isopropyl $\beta$-D-1-thiogalactopyranoside $(0.5 \mathrm{mM})$. Bacterial cultivation was subsequently continued at $16^{\circ} \mathrm{C}$ and $200 \mathrm{rpm}$ for 16 to 24 hours (for Stim $1 \mathrm{C}$-tail in pGEX6p1) or 2 hours (for Bin2 in pETM11). Cells were harvested by centrifugation at $6000 \mathrm{rpm}$ for 15 minutes at $4^{\circ} \mathrm{C}$ (Beckman Coulter centrifuge, rotor type JA-10) and cell pellets (up to $20 \mathrm{~g} / 500 \mathrm{~mL}$ expression culture) were flash-frozen and stored at $-80^{\circ} \mathrm{C}$. Generally, protein production was controlled by 1D SDS-PAGE and Western blotting.

\section{Protein purification}

STIM1 C-tail. The harvested cell pellets were resuspended in chilled lysis buffer (50 mM Tris- $\mathrm{HCl}, 150 \mathrm{mM} \mathrm{NaCl}, \mathrm{pH}$ 8, supplemented with protease and phosphatase inhibitors) to a final concentration of $0.2 \mathrm{~g} / \mathrm{mL}$. Subsequent cell lysis was performed by sonification $(4 \times 30$ seconds) on ice. The cell lysate was centrifuged at 16,000 rpm for 1 hour at $4^{\circ} \mathrm{C}$ (Beckman Coulter centrifuge, rotor type JA 25.50), and the resulting supernatant was incubated with Ni-NTA agarose $(0.5 \mathrm{~mL}$ per $1 \mathrm{~g}$ starting material, preequilibrated with lysis buffer) under constant stirring for 1 hour at $4^{\circ} \mathrm{C}$. After incubation, the Ni-NTA agarose was transferred to an Econo-Column $2.5 \times 20 \mathrm{~cm}$ column body (Bio-Rad) and washed intensively with at least $150 \mathrm{~mL}$ lysis buffer before the bound protein was eluted with $25 \mathrm{~mL}$ lysis buffer supplemented with elution buffer ( $50 \mathrm{mM}$ Tris- $\mathrm{HCl}, 150 \mathrm{mM} \mathrm{NaCl}, \mathrm{pH}$ 8, supplemented with $250 \mathrm{mM}$ imidazole). The protein was concentrated using Amicon Ultra centrifugal filter units to a volume of $1 \mathrm{~mL}$ and further purified using size exclusion chromatography with a Superdex 200 Increase 10/300 GL column pre-equilibrated with fast protein liquid chromatography (FPLC) buffer (50 mM Tris- $\mathrm{HCl}, 150 \mathrm{mM} \mathrm{NaCl}, \mathrm{pH} 8.0$ ) on an Äkta pure 25 system (GE Healthcare). Fractions were collected (1 $\mathrm{mL}$ volume per fraction) and stored at $4^{\circ} \mathrm{C}$. Success of the purification was verified using 1D SDS-PAGE and Western blotting. Afterward, fractions containing the pure protein were pooled and concentrated using Amicon Ultra centrifugal filter units, and the protein concentration was determined using a NanoDrop device (Thermo Fisher Scientific). The protein was stored at $-80^{\circ} \mathrm{C}$ until further usage.

BIN2. The harvested cell pellets were resuspended in buffer A (50 mM HEPES, $150 \mathrm{mM} \mathrm{NaCl}$, 5\% glycerol, $\mathrm{pH}$ 7.6; $10 \mathrm{~mL}$ per g pellet). To separate the soluble and insoluble protein fraction, cells were lysed mechanically by sonification $(4 \times 30$ seconds) on ice. The cell lysate was centrifuged at $16,000 \mathrm{rpm}$ for 1 hour at $4^{\circ} \mathrm{C}$ (Beckman Coulter centrifuge, rotor type JA 25.50). The supernatant was subsequently subjected to immobilized metal affinity chromatography on an FPLC system (Äkta pure $25 \mathrm{M}, \mathrm{GE}$ Healthcare) with an attached HisTrap HP $5 \mathrm{~mL}$ column (GE Healthcare). The sample was loaded completely onto the column, which was pre-equilibrated in buffer A, then the column was washed with 20 column volumes ( $20 \mathrm{CVs}$ ) of buffer A. There were 2 washing steps with $5 \%$ and $12 \%$ of buffer B (50 mM HEPES, $150 \mathrm{mM} \mathrm{NaCl}, 5 \%$ glycerol, $400 \mathrm{mM}$ imidazole, $\mathrm{pH}$ 7.6). Proteins were eluted using $100 \%$ elution buffer B. Samples of all purification steps were analyzed by SDS-PAGE, and the eluate fractions that contained the target protein were pooled. The purified protein was concentrated using Amicon Ultra centrifugal filter units, and protein concentration was determined using a NanoDrop device (Thermo Fisher Scientific). In general, the purified proteins were flash-frozen and stored until further usage at $-80^{\circ} \mathrm{C}$, and the purification procedure was monitored by 1D SDS-PAGE and Western blotting.

\section{CST pulldown assay}

To determine whether the interaction between BIN2 and STIM1 was direct, GST pulldown assays with purified recombinant proteins were performed. For this purpose, $2.5 \mu \mathrm{M}$ GST-tagged STIM1 C-tail in 200 $\mu \mathrm{L}$ pulldown buffer (1× PBS supplemented with $5 \mathrm{mM}$ DTT) were coupled to $20 \mu \mathrm{L}$ pre-equilibrated Pierce glutathione agarose beads (Thermo Fisher Scientific) for 2 hours at $4^{\circ} \mathrm{C}$ under constant rotation. Afterward, the beads were washed 3 times with $400 \mu \mathrm{L}$ pulldown buffer (centrifugation $500 \mathrm{~g}, 1$ minute, $4^{\circ} \mathrm{C}$ ). Subsequently, increasing concentrations of recombinant BIN2 (starting with $2.5 \mu \mathrm{M}$ up to 500 $\mu \mathrm{M})$ in $200 \mu \mathrm{L}$ pulldown buffer were incubated with the agarose beads for 2 hours at $4^{\circ} \mathrm{C}$ under constant rotation. Afterward, the beads were washed 3 times with $400 \mu \mathrm{L}$ pulldown buffer (centrifugation $500 g, 1$ minute, $4^{\circ} \mathrm{C}$ ), supplemented with $5 \mu \mathrm{L} 4 \times$ Laemmli buffer, and samples were analyzed by silver staining.

\section{Immobilization of purified recombinant STIM1 C-tail on Affi-Gel 10}

Protein coupling to Affi-Gel 10 (Bio-Rad) was performed under aqueous conditions according to the manufacturer's instructions. For each sample, an Affi-Gel 10 slurry volume of $1 \mathrm{~mL}$ was transferred to an 
Econo Chromatography Column $(2.5 \times 20 \mathrm{~cm})$ and washed with $3 \mathrm{CVs}$ deionized water $\left(4^{\circ} \mathrm{C}\right)$ at gravity flow. Upon addition of the protein solution (at least $0.5 \mathrm{~mL}$ per $\mathrm{mL}$ gel), binding was performed by headover-tail rotation at $4^{\circ} \mathrm{C}$ for 4 hours. The column was subsequently washed with $1 \mathrm{CV}$ PBS ( $\mathrm{pH}$ 7.4), and remaining free active ester was blocked by incubating in $1 \mathrm{M}$ ethanolamine- $\mathrm{HCl}$ solution ( $\mathrm{pH}$ 8.0, at least $0.1 \mathrm{~mL}$ per $\mathrm{mL}$ gel) at $4^{\circ} \mathrm{C}$ for 1 hour. Finally, the resulting affinity matrix was washed with $3 \mathrm{CVs}$ of ice-cold deionized water and stored in an aqueous solution of $0.2 \%(\mathrm{w} / \mathrm{v})$ sodium azide $(\mathrm{pH} 7.4)$ at $4^{\circ} \mathrm{C}$.

\section{Interactome screening of native platelet lysates}

The generated STIM1 C-tail affinity column was washed twice in icecold deionized water and then pre-equilibrated in $10 \mathrm{CVs}$ ice-cold PBS $(\mathrm{pH} 7.4)$ at $4^{\circ} \mathrm{C}$. Native platelet lysate was added (at least $10 \mathrm{mg}$ per $\mathrm{mL}$ affinity support), the remaining column volume was filled with platelet lysis buffer, and incubation was performed by head-over-tail rotation overnight at $4^{\circ} \mathrm{C}$. In parallel, to also evaluate the impact of unspecific protein binding to the affinity column/material, an equal volume of native platelet lysate was incubated with uncoupled Affi-Gel 10 matrix (i.e., control column, Ctrl). The next day, the supernatant was discarded by gravity flow, the bead material was washed with 10 CVs PBS, and bound proteins were eluted by applying a $\mathrm{pH}$ gradient of $10 \mathrm{mM}$ glycine/HCl pH 6.0, pH 5.0, pH 4.0, pH 3.0, and pH 2.0. For each pH step, an elution volume of $3 \mathrm{CVs}$ was collected. Immediately after elution, $\mathrm{pH}$ values were adjusted to $\mathrm{pH} 7.0$ by addition of an appropriate volume of $1 \mathrm{M}$ Tris buffer, and the total volume of the individual $\mathrm{pH}$ fractions was reduced to $1 \mathrm{~mL}$ in an SPD $111 \mathrm{~V}$ Speed $\operatorname{Vac}\left(35^{\circ} \mathrm{C}\right)$. The resulting elution samples were either processed further or stored at $-80^{\circ} \mathrm{C}$.

\section{Mass spectrometry analysis of the STIM1 interactome}

1D SDS-PAGE of elution samples, gel silver staining, tryptic digestion of differential protein spots, and sample preparation for subsequent mass spectrometry (MS) analysis were performed as published previously (55). Tryptic peptides were separated on an Ultimate nano-HPLC system (Dionex) as reported previously (56) and directly eluted into the online electrospray ion source (ESI) of a Qtrap 4000 (Applied Biosystems) and an LTQ XL (Thermo Fisher Scientific) mass analyzer. Full MS survey scans from 350 to $2000 \mathrm{~m} / z$ were acquired, and the 3 most intense peaks were subjected to collision-induced dissociation MS/MS. Mass spectra were recorded via the Analyst 1.4 (QTrap) and the Xcalibur 2.1.0 (LTQ) operating software including the mascot.dll 1.6b5 (QTrap) and the extract_msn (LTQ) plug-in for conversion of LC-MS raw data into Mascot generic format. Applying the Mascot search algorithm for MS/MS spectra (Mascot Daemon 2.2.02 software platform and Mascot server version 2.2.04), the generated data were searched against the UniProtKB/ Swiss-Prot database (www.uniprot.org; accessed November, 2009; human subset), with Mascot parameters being set as published previously (56). All MS/MS spectra with a minimum Mascot score of 34 ( $P$ value of 0.05 ) were taken into consideration for further interpretation and were additionally validated manually.

\section{BIN2 pulldown}

Platelet lysates from Bin $2^{f / f f, P f 4 C r e}$ mice $\left(\sim 1 \times 10^{8}\right.$ platelets in $\left.50 \mu \mathrm{L}\right)$ were mixed with $30 \mu \mathrm{g}$ of BIN2-HIS ( $9 \mu \mathrm{M}$ final concentration) for $5 \mathrm{~min}$ utes at room temperature, followed by a protein purification step using Ni-NTA agarose (QIAGEN). Next, $40 \mu \mathrm{L}$ of Ni-NTA agarose was filled into a spin column and washed with buffer A. The lysate was incu- bated with the beads for 10 minutes before it was slowly centrifuged through the column $(500 \mathrm{~g})$. After 3 washing steps, the bound protein was eluted using buffer B. Each fraction was subsequently analyzed using SDS-PAGE and Western blot.

\section{BIN2 IP}

Platelet lysate $\left(400 \mu \mathrm{L}\right.$ of $1 \times 10^{6}$ platelets $\left./ \mu \mathrm{L}\right)$ were incubated on Protein GE Sepharose (GE Healthcare), which was preincubated with $3 \mu \mathrm{g}$ of the indicated BIN2 antibodies, for 2 hours at $4^{\circ} \mathrm{C}$. After extensive washing, the beads were incubated with Western blot sample buffer for 5 minutes at $95^{\circ} \mathrm{C}$, followed by centrifugation. The protein-containing supernatant was analyzed by Western blot. The following BIN2 antibodies were used: anti-BIN2 (Proteintech, 14245-1-AB), anti-BIN2 (N-terminal, Abcam, 175482), 12E1 (lab-made, rat IgG2Aк), and 2C11 (lab-made, rat IgG2A).

\section{Fluorescence polarization assay}

Fluorescence polarization assay was performed to assess the affinity of the STIM1-BIN2 interaction. The GST tag of the STIM1 C-tail was cut by $3 \mathrm{c}$ protease cleavage ( $67 \mu \mathrm{g} 3 \mathrm{c}$ protease per $1 \mathrm{mg}$ STIM $1 \mathrm{C}$-tail), and the cleaved tag was captured with glutathione agarose while the cleaved STIM1 C-tail was collected in the eluate. The GST-free STIM1 C-tail protein was labeled with BDP FL NHS-Ester (Lumiprobe) in TBS overnight at $4^{\circ} \mathrm{C}$ in a ratio of 1.0:0.8, resulting in a degree of labeling of 0.7.

Recombinant BIN2 was added at concentrations ranging from 0 $\mu \mathrm{M}$ to $400 \mu \mathrm{M}$ to $1 \mu \mathrm{M}$ STIM1-BDP FL in a 384 -well plate (Greiner) and fluorescence polarization was measured in triplicate on a CLARIOstar (BMG Labtech) plate reader (excitation: 482-16 nm; emission: 530-40 nm). Data analysis and curve fitting were performed with GraphPad Prism.

\section{Human platelet purification and lysis}

Blood was taken from consenting, healthy, drug-free volunteers. Platelets were purified as described previously (57). Platelet pellets (aliquot volume: $100 \mu \mathrm{L}$ ) were suspended in chilled native platelet lysis buffer (100 mM Tris-HCl, 2 mM EDTA, 2\% [v/v] Triton X-100, protease and phosphatase inhibitors [Complete Mini Protease- and PhosSTOP Phosphatase Inhibitor, Roche]) and lysed by sonication in a Sonorex RK 52 ultrasonic bath (5 seconds at 70\% maximum power rate, 10 intervals). Each interval was followed by 3 seconds of vortexing and chilling on ice. The resulting lysate was cleared from remaining cellular debris by 30 minutes of centrifugation at $20,000 \mathrm{~g}\left(4^{\circ} \mathrm{C}\right)$. The protein content of the supernatant was subsequently quantified by BCA assay.

\section{Mouse platelet preparation}

Washed platelets were prepared as described previously (58). Briefly, mice were anesthetized using isoflurane and bled into $300 \mu \mathrm{L}$ heparin (20 U/mL in TBS, Ratiopharm). The blood was centrifuged twice at $300 \mathrm{~g}$ for 6 minutes to obtain platelet-rich plasma (PRP). PRP was supplemented with $0.02 \mathrm{U} / \mathrm{mL}$ apyrase (MilliporeSigma, A610) and 0.1 $\mu \mathrm{g} / \mathrm{mL} \mathrm{PGI}_{2}$ (MilliporeSigma, P6188), and platelets were pelleted by centrifugation at $800 \mathrm{~g}$ for 5 minutes and washed twice with Tyrode's HEPES buffer (134 mM NaCl, $0.34 \mathrm{mM} \mathrm{Na}_{2} \mathrm{HPO}_{4}, 2.9 \mathrm{mM} \mathrm{KCl}, 12 \mathrm{mM}$ $\mathrm{NaHCO}_{3}, 5 \mathrm{mM}$ HEPES, $5 \mathrm{mM}$ glucose, $0.35 \%$ BSA, pH 7.4) containing $0.02 \mathrm{U} / \mathrm{mL}$ apyrase and $0.1 \mu \mathrm{g} / \mathrm{mL} \mathrm{PGI}_{2}$. The platelets were allowed to rest for 30 minutes before experiments. 


\section{Immunoblotting}

Western blot analysis was performed as described previously (58). Untreated or activated platelets were lysed at the respective time points, separated by SDS-PAGE, and blotted on a PVDF membrane, on which the proteins were detected using anti-BIN2 antibody (Proteintech, 14245-1-AB), anti-STIM1 antibody (Cell Signaling Technology, 4916), anti-IP ${ }_{3}$ R antibody (Merck-Millipore, 07-1210), anti-RhoA (Cytoskeleton, ARH04), and anti-GAPDH (MilliporeSigma, G9545). Human protein lysates were purchased from ProSci. Silver staining of the SDS-PAGE was performed using the SilverQuest Silver Staining Kit (Life Technologies) according to the manufacturer's instructions.

\section{Blood parameter measurements}

The blood parameters were measured using a ScilVET analyzer as previously described (59).

\section{Flow cytometry}

Flow cytometry to determine glycoprotein expression, integrin activation, and P-selectin exposure was performed as previously described (58). In short, heparinized whole blood was diluted 1:20 in Tyrode's HEPES buffer. For activation studies, the blood samples were washed twice in Tyrode's HEPES and finally resuspended in Tyrode's HEPES buffer containing $\mathrm{Ca}^{2+}$ before agonist addition. The samples were incubated with saturating amounts of fluorophore-conjugated mAbs for 15 minutes at room temperature, and analyzed on a FACSCalibur (BD Biosciences). The reaction was stopped by addition of $500 \mu \mathrm{L}$ PBS buffer, and samples were directly analyzed on a FACSCalibur.

To measure PS exposure on the platelet surface, washed platelets $\left(5 \times 10^{4}\right.$ platelets $\left./ \mu \mathrm{L}\right)$ in Tyrode's buffer with $2 \mathrm{mM} \mathrm{Ca}^{2+}$ were incubated with the indicated agonists for 15 minutes at $37^{\circ} \mathrm{C}$ in the presence of saturating amounts of DyLight 488-coupled annexin A5 (purified and labeled in our laboratory). The reaction was stopped by addition of $500 \mu \mathrm{L}$ PBS buffer with $2 \mathrm{mM} \mathrm{Ca}{ }^{2+}$. Analysis was performed on a FACSCalibur.

\section{Aggregometry}

Washed platelets were prepared as described previously (58). For aggregometry, washed platelets $\left(160 \mu \mathrm{L}\right.$ of $1.5 \times 10^{5}$ platelets $\left./ \mu \mathrm{L}\right)$ were analyzed in the absence (thrombin) or presence (all other agonists) of $70 \mu \mathrm{g} / \mathrm{mL}$ human fibrinogen. Light transmission was recorded on a 4-channel aggregometer (Fibrintimer, APACT) for 10 minutes and expressed in arbitrary units, with buffer representing $100 \%$ light transmission.

\section{Spreading}

Spreading experiments were performed as described previously (58). Briefly, thrombin-stimulated platelets were allowed to spread on fibrinogen-coated coverslips for the indicated time points. Representative interference contrast images (Zeiss Axiovert 200 inverted microscope) were acquired, and the different spreading phases were determined for quantification.

\section{Platelet lifespan}

Platelet lifespan was measured by injecting $5 \mu \mathrm{g}$ of DyLight 488 -conjugated anti-GPIX derivative. The percentage of circulating labeled platelets was measured daily by flow cytometry using $50 \mu \mathrm{L}$ whole blood $(58,60)$.

\section{Intracellular calcium measurements}

The measurements were performed as previously described (61). In brief, washed platelets were loaded with $2.5 \mu \mathrm{M}$ Fura2/AM (Life Technologies, F1221) in the presence of $0.2 \mu \mathrm{g} / \mathrm{mL}$ pluronic F-127 (Life Technologies, P6867) for 30 minutes at $37^{\circ} \mathrm{C}$. After 1 washing step, the platelets were resuspended in HBSS, and the emission was measured at $509 \mathrm{~nm}$ under stirring conditions in a fluorimeter (LS55, PerkinElmer) with excitation alternating between $340 \mathrm{~nm}$ and 380 $\mathrm{nm}$. After each measurement, calibration was performed with Triton $\mathrm{X}-100$ and EGTA.

\section{IP ELISA}

To determine IP levels, a measure of $\mathrm{IP}_{3}$ production in platelets, a commercially available ELISA kit (Cisbio) was used. The platelets were purified from mouse blood as described above, and the last washing step was performed with phosphate-free Tyrode's. The platelet count was adjusted to $700,000 / \mu \mathrm{L}$ using phosphate-free Tyrode's containing $2 \mathrm{mM} \mathrm{Ca}^{2+}$ and $50 \mathrm{mM}$ lithium chloride. After 5 minutes of incubation with indomethacin $(10 \mu \mathrm{M})$, apyrase $(2 \mathrm{U} / \mathrm{mL})$, and EDTA ( $5 \mathrm{mM})$, the platelets were activated with the indicated agonist for 15 minutes, followed by lysis for 30 minutes at $37^{\circ} \mathrm{C}$ using the lysis reagent provided with the kit. ELISA was performed according to the manufacturer's manual.

\section{Flow chamber}

Platelet adhesion under flow was measured by perfusing murine whole blood on glass coverslips coated with $200 \mu \mathrm{g} / \mathrm{mL}$ fibrillar type I collagen (Takeda). The slides were coated overnight at $37^{\circ} \mathrm{C}$ and then blocked with $1 \%$ BSA/PBS for 1 hour at room temperature. Then, 700 $\mu \mathrm{L}$ mouse blood was collected in $300 \mu \mathrm{L}$ heparin (20 U/mL in TBS), and the platelets were labeled with a DyLight 488 -conjugated antiGPIX Ig derivate $(0.2 \mu \mathrm{g} / \mathrm{mL})$ for 5 minutes at $37^{\circ} \mathrm{C}$. The blood was perfused over the coverslip at a shear rate of $1700 / \mathrm{s}$. Phase-contrast and fluorescence images were acquired using a Leica DMI6000B inverted microscope $(\times 63 / 1.3$ glycerol HCX PL APO objective) equipped with a Leica DFC 360 FX camera and analyzed using Image (NIH).

For measurement of procoagulant activity in this setting, experiments were performed as described above, with the Tyrode's buffer supplemented with additional $5 \mathrm{U} / \mathrm{mL}$ heparin to prevent coagulation. To detect PS-exposing platelets, Tyrode's containing $0.25 \mu \mathrm{g} /$ $\mathrm{mL}$ annexin A5-DyLight 488 was perfused over the adherent platelets, followed by a washing step before bright-field and fluorescence images were taken. Procoagulant activity was defined as the ratio of surface coverage of PS-exposing platelets to the total surface covered by platelets.

\section{Tail bleeding time and in vivo thrombus formation}

Tail bleeding time and in vivo thrombus formation studies in the mechanically injured abdominal aorta were performed as described elsewhere (58).

\section{tMCAO model}

tMCAO was performed as described previously (45). Briefly, a thread was advanced into the middle cerebral artery to inhibit blood flow for 60 minutes, followed by removal of the filament to allow reperfusion. Then, 24 hours later, infarct sizes were analyzed in brain sections by TTC staining. Histology and IHC were performed according 
to standard procedures (62). Cryoembedded coronal brain sections $(2 \mathrm{~mm})$ were cut into $10-\mu \mathrm{m}$-thick slices. Every tenth slice was used for evaluation. The following antibodies were used: $\mathrm{mAb}$ anti-CD11b (MCA711, Serotec) and anti-Ly-6B (MCA771G, Bio-Rad). For quantification of occluded microvessels, brain sections were stained with hematoxylin and eosin.

\section{dSTORM sample preparation}

Spread platelets on fibrinogen and resting platelets on glycine were fixed with $3 \%$ glyoxal solution (63), quenched with $0.1 \% \mathrm{NaBH}_{4}$ (ICN Biomedicals), permeabilized with $0.25 \%$ Triton X-100 (Electran), and blocked in a 5\% BSA solution in PBS. Murine platelets were stained with Alexa Fluor 647-coupled anti-BIN2 antibody (generated and modified in our laboratory as described previously (64). Antibody specificity was tested by Western blotting, displaying 1 band in WT and no band in Bin2 $2^{f / f, P f 4 C r e}$ platelet lysates); human platelets were stained with Alexa Fluor 532-coupled anti-BIN2-antibody (Proteintech, 14245-1-AB) and Alexa Fluor 647-coupled anti-STIM1-antibody (Santa Cruz Biotechnology, sc-166840).

\section{dSTORM microscopy}

One-color $d$ STORM samples were imaged on a widefield setup based on an inverted microscope (Olympus IX-71) equipped with an oil immersion objective ( $\times 60$ original magnification, NA 1.45; Olympus). The dye was excited with a diode laser at $641 \mathrm{~nm}$ at irradiation intensities between 2 and $10 \mathrm{~kW} / \mathrm{cm}^{2}$ (Cube 640-100C, Coherent). Emission light was separated from the excitation light using a dichroic mirror (ZT 405/514/635rpc, Chroma Technology Corp.), spectrally filtered by a bandpass filter (Em01-R442/514/647-25; Semrock) and projected onto an EMCCD camera chip (iXon DU-897, Andor). Imaging was performed with an exposure time of $20 \mathrm{~ms}$ for 15,000 frames in photo-switching buffer containing $100 \mathrm{mM} \beta$-mercaptoethylamine, $\mathrm{pH}$ 7.4 without oxygen scavenger (35).

Two-color $d$ STORM was performed on the same setup described above. Alexa Fluor 647 and Alexa Fluor 532 were excited with the appropriate laser systems (Genesis MX639 and MX514, Coherent) at irradiation intensities between 2 and $10 \mathrm{~kW} / \mathrm{cm}^{2}$. Emission light was separated from the excitation light using a dichroic mirror (ZT 405/514/635rpc, Chroma Technology Corp.), spectrally filtered by a bandpass filter (Em01-R442/514/647-25, Semrock), transmitted by a dichroic beam splitter (ZT 405/514/635rpc, Chroma Technology Corp.) and respective emission filters (Brightline HC 679/41, Brightline HC 582/75, Semrock), before being projected on 2 EMCCD camera chips (iXon Ultra DU-897, Andor). Imaging was done with an exposure time of $20 \mathrm{~ms}$ for 15,000 frames (red dye) and 30,000 frames (green dye) in photo-switching buffer containing $100 \mathrm{mM} \beta$-mercaptoethylamine, $\mathrm{pH}$ 6.9, as previously described (56). Fluorescent beads (TetraSpeck) were imaged in both channels over the whole ROI to later correct for chromatic aberrations. Spread platelets were imaged by total internal reflection fluorescence (TIRF) illumination; resting platelets were measured by epifluorescence (EPI). dSTORM images were reconstructed using the open source software rapidSTORM 3.3 (65); matrices for chromatic aberration corrections were done with the bUnwarpJ plugin in Fiji (58).

The neighbor analysis was performed with MATLAB (MathWorks Inc.) along the neighbor density analysis concepts described by Malkusch et al. (50). For the analysis, only the localization data of STIM1 and BIN2 within the region of interest of a single platelet was considered, while in the case of activated platelets only the inner platelet region was considered for STIM1 localizations (Figure 4, C and D). The distance-dependent BIN2 density was normalized to the total number of STIM1 localizations in the platelet region of interest and the average BIN2 density at distances between 400 and $500 \mathrm{~nm}$. As controls, random distributions of localization data were generated, with densities corresponding to the values of the experimental data.

\section{Statistics}

Data are represented as mean \pm SD. $P$ values were calculated with the 2-tailed Student's $t$ test, Mann-Whitney $U$ test, or Kruskal-Wallis test followed by Dunn's multiple-comparisons post hoc test, as indicated in the figure legend. In case of frequency analysis of occluded and nonoccluded vessels in the aorta injury model, Fisher's exact test was used to compare groups. For statistical analysis, GraphPad Prism 6 software was used. $P$ values of less than 0.05 were considered statistically significant $\left({ }^{*} P<0.05,{ }^{* *} P<0.01,{ }^{* * *} P<0.001\right)$.

\section{Study approval}

Animal studies were approved by the district government of Lower Franconia (Bezirksregierung Unterfranken). The mice were mixed sex, aged between 8 and 12 weeks. Human platelet samples were anonymized waste materials from studies approved by the Ethics Committee of the Medical Faculty of the University of Würzburg. All human blood donors gave written, fully informed consent.

\section{Author contributions}

JV, CK, SB, MP, IS, TV, MM, JP, MKS, KH, TP, and AB performed experiments and analyzed data. AS, DS, GS, AB, HSH, KGH, MS, and $\mathrm{BN}$ analyzed data from experiments, discussed results, and provided scientific input throughout the study. JV, MP, TV, AB, and $\mathrm{BN}$ wrote the manuscript with input from all the authors. BN conceived the study and designed research. Julia Volz has been the lead author and lead scientist in this project from the beginning. During the course of revision, Charly Kusch contributed a significant amount of microscopy data that was essential for the final acceptance of the manuscript and therefore he was named as a second lead author. Julia Volz remained in the first position because of her continuing involvement in the project and her long-lasting contributions.

\section{Acknowledgments}

This study was supported by the Deutsche Forschungsgemeinschaft (SFB/TR 240, project number 374031971, A07 to BN and MS, B02 to GS, B06 to KGH, MS and DS, Z02 to AS, and grant NI $556 / 11-2$ to $\mathrm{BN}$ ) and by the European Union (Thrombo-Inflame, EFRE-Europäischer Fonds für regionale Entwicklung, Bavaria). We thank Sylvia Hengst and Birgit Midloch for excellent technical assistance and Katharina Remer for support with documentation and management of animal experimentation and welfare. We also thank Lars Schönemann and Michelle Endres from the recombinant protein expression facility of the Rudolf Virchow Center for purification of recombinant BIN2 and STIM1. JV was supported by a grant of the German Excellence Initiative to the Graduate School of Life Sciences, University of Würzburg. AS is supported by the 
Ministerium für Kultur und Wissenschaft des Landes NordrheinWestfalen, the Regierende Bürgermeister von Berlin inkl. Wissenschaft und Forschung, and the Bundesministerium für Bildung und Forschung.

Address correspondence to: Bernhard Nieswandt, Institute of Experimental Biomedicine I, University Hospital Würzburg and
Rudolf Virchow Center, University of Würzburg, Josef-SchneiderStrausse 2, 97080 Würzburg, Germany, Phone: 49.931.31.80405; Email: bernhard.nieswandt@virchow.uni-wuerzburg.de.

AB's present address is Walther-Straub Institute of Pharmacology and Toxicology, Ludwig-Maximilians-Universität München, Munich, Germany.
1. Jackson SP. Arterial thrombosis--insidious, unpredictable and deadly. Nat Med. 2011;17(11):1423-1436.

2. Bhatt DL. Intensifying platelet inhibition--navigating between Scylla and Charybdis. $N$ Engl J Med. 2007;357(20):2078-2081.

3. Jackson SP. The growing complexity of platelet aggregation. Blood. 2007;109(12):5087-5095.

4. Stoll G, Nieswandt B. Thrombo-inflammation in acute ischaemic stroke - implications for treatment. Nat Rev Neurol. 2019;15(8):473-481.

5. Stegner D, Nieswandt B. Platelet receptor signaling in thrombus formation. J Mol Med (Berl). 2011;89(2):109-121.

6. Mammadova-Bach E, Nagy M, Heemskerk JWM, Nieswandt B, Braun A. Store-operated calcium entry in thrombosis and thrombo-inflammation. Cell Calcium. 2019;77:39-48.

7. Grosse J, et al. An EF hand mutation in Stim1 causes premature platelet activation and bleeding in mice. JClin Invest. 2007;117(11):3540-3550.

8. Bergmeier W, Oh-Hora M, McCarl CA, Roden RC, Bray PF, Feske S. R93W mutation in Orai1 causes impaired calcium influx in platelets. Blood. 2009;113(3):675-678.

9. Varga-Szabo D, et al. The calcium sensor STIM1 is an essential mediator of arterial thrombosis and ischemic brain infarction. J Exp Med. 2008;205(7):1583-1591.

10. Braun A, et al. Orai1 (CRACM1) is the platelet SOC channel and essential for pathological thrombus formation. Blood. 2009;113(9):2056-2063.

11. Liou J, et al. STIM is a $\mathrm{Ca}^{2+}$ sensor essential for $\mathrm{Ca}^{2+}$-store-depletion-triggered $\mathrm{Ca}^{2+}$ influx. Curr Biol. 2005;15(13):1235-1241.

12. Luik RM, Wu MM, Buchanan J, Lewis RS. The elementary unit of store-operated $\mathrm{Ca}^{2+}$ entry: local activation of CRAC channels by STIM1 at ER-plasma membrane junctions. J Cell Biol. 2006;174(6):815-825.

13. Wu MM, Buchanan J, Luik RM, Lewis RS. $\mathrm{Ca}^{2+}$ store depletion causes STIM1 to accumulate in ER regions closely associated with the plasma membrane. J Cell Biol. 2006;174(6):803-813.

14. Zhang SL, et al. STIM1 is a $\mathrm{Ca}^{2+}$ sensor that activates CRAC channels and migrates from the $\mathrm{Ca}^{2+}$ store to the plasma membrane. Nature. 2005;437(7060):902-905.

15. Xu P, Lu J, Li Z, Yu X, Chen L, Xu T. Aggregation of STIM1 underneath the plasma membrane induces clustering of Orai1. Biochem Biophys Res Commun. 2006;350(4):969-976.

16. Thillaiappan NB, Chavda AP, Tovey SC, Prole $\mathrm{DL}$, Taylor $\mathrm{CW} . \mathrm{Ca}^{2+}$ signals initiate at immobile $\mathrm{IP}_{3}$ receptors adjacent to ER-plasma membrane junctions. Nat Commun. 2017;8(1):1505.

17. Sampieri A, Santoyo K, Asanov A, Vaca L. Association of the IP3R to STIM1 provides a reduced intraluminal calcium microenvironment, resulting in enhanced store-operated calcium entry. Sci Rep. 2018;8(1):13252.

18. Feng JM, et al. Golli protein negatively regulates store depletion-induced calcium influx in T cells. Immunity. 2006;24(6):717-727.

19. Palty R, Raveh A, Kaminsky I, Meller R, Reuveny E. SARAF inactivates the store operated calcium entry machinery to prevent excess calcium refilling. Cell. 2012;149(2):425-438.

20. Srikanth S, Jew M, Kim KD, Yee MK, Abramson J, Gwack Y. Junctate is a $\mathrm{Ca}^{2+}$-sensing structural component of Orail and stromal interaction molecule 1 (STIM1). Proc Natl Acad Sci U S A. 2012;109(22):8682-8687.

21. Berridge MJ, Bootman MD, Roderick HL. Calcium signalling: dynamics, homeostasis and remodelling. Nat Rev Mol Cell Biol. 2003;4(7):517-529.

22. Groschner K, Shrestha N, Fameli N. Non-orai partners of STIM proteins: role in ER-PM communication and $\mathrm{Ca}^{2+}$ signaling. In: Kozak JA, Putney JW Jr., eds. Calcium Entry Channels in Non-Excitable Cells. CRC Press/Taylor \& Francis; 2018:177-196.

23. Stanishneva-Konovalova TB, Derkacheva NI, Polevova SV, Sokolova OS. The role of BAR domain proteins in the regulation of membrane dynamics. Acta Naturae. 2016;8(4):60-69.

24. Carman PJ, Dominguez R. BAR domain proteins-a linkage between cellular membranes, signaling pathways, and the actin cytoskeleton. Biophys Rev. 2018;10(6):1587-1604.

25. Ren G, Vajjhala P, Lee JS, Winsor B, Munn AL. The BAR domain proteins: molding membranes in fission, fusion, and phagy. Microbiol Mol Biol Rev. 2006;70(1):37-120.

26. Sakamuro D, Elliott KJ, Wechsler-Reya R, Prendergast GC. BIN1 is a novel MYC-interacting protein with features of a tumour suppressor. Nat Genet. 1996;14(1):69-77.

27. Hong TT, et al. BIN1 localizes the L-type calcium channel to cardiac T-tubules. PLOS Biol. 2010;8(2):e1000312.

28. Hong T, et al. Cardiac BIN1 folds T-tubule membrane, controlling ion flux and limiting arrhythmia. Nat Med. 2014;20(6):624-632.

29. Simionescu-Bankston A, et al. The N-BAR domain protein, Bin3, regulates Rac1- and Cdc42-dependent processes in myogenesis. Dev Biol. 2013;382(1):160-171.

30. Ge K, Prendergast GC. Bin2, a functionally nonredundant member of the BAR adaptor gene family. Genomics. 2000;67(2):210-220.

31. Sanchez-Barrena MJ, et al. Bin2 is a membrane sculpting N-BAR protein that influences leucocyte podosomes, motility and phagocytosis. PLoS One. 2012;7(12):e52401.

32. Baba Y, Nishida K, Fujii Y, Hirano T, Hikida M,
Kurosaki T. Essential function for the calcium sensor STIM1 in mast cell activation and anaphylactic responses. Nat Immunol. 2008;9(1):81-88.

33. Gwack Y, et al. Hair loss and defective T- and B-cell function in mice lacking ORAI1. Mol Cell Biol. 2008;28(17):5209-5222.

34. Münzer P, et al. CK2 $\beta$ regulates thrombopoiesis and $\mathrm{Ca}^{2+}$-triggered platelet activation in arterial thrombosis. Blood. 2017;130(25):2774-2785.

35. van de Linde S, et al. Direct stochastic optical reconstruction microscopy with standard fluorescent probes. Nat Protoc. 2011;6(7):991-1009.

36. Heilemann M, et al. Subdiffraction-resolution fluorescence imaging with conventional fluorescent probes. Angew Chem Int Ed Engl. 2008;47(33):6172-6176.

37. Weber K, Rathke PC, Osborn M. Cytoplasmic microtubular images in glutaraldehyde-fixed tissue culture cells by electron microscopy and by immunofluorescence microscopy. Proc Natl Acad SciUS A. 1978;75(4):1820-1824

38. Durrant TN, van den Bosch MT, Hers I. Integrin $\alpha_{\mathrm{IL}} \beta_{3}$ outside-in signaling. Blood. 2017;130(14):1607-1619.

39. Gilio K, et al. Roles of platelet STIM1 and Orai1 in glycoprotein VI- and thrombin-dependent procoagulant activity and thrombus formation. J Biol Chem. 2010;285(31):23629-23638.

40. Grüner S, et al. Anti-glycoprotein VI treatment severely compromises hemostasis in mice with reduced alpha2beta1 levels or concomitant aspirin therapy. Circulation. 2004;110(18):2946-2951.

41. Inoue O, Suzuki-Inoue K, Dean WL, Frampton J, Watson SP. Integrin alpha2beta1 mediates outside-in regulation of platelet spreading on collagen through activation of Src kinases and PLCgamma2. J Cell Biol. 2003;160(5):769-780.

42. Dutting S, et al. Growth factor receptor-bound protein 2 contributes to (hem)immunoreceptor tyrosine-based activation motif-mediated signaling in platelets. Circ Res. 2014;114(3):444-453.

43. Cherpokova D, et al. SLAP/SLAP2 prevent excessive platelet (hem)ITAM signaling in thrombosis and ischemic stroke in mice. Blood. 2015;125(1):185-194.

44. Dong M, Zheng N, Ren LJ, Zhou H, Liu J. Increased expression of STIM1/Orai1 in platelets of stroke patients predictive of poor outcomes. Eur J Neurol. 2017;24(7):912-919.

45. Kleinschnitz C, Pozgajova M, Pham M, Bendszus M, Nieswandt B, Stoll G. Targeting platelets in acute experimental stroke: impact of glycoprotein Ib, VI, and IIb/IIIa blockade on infarct size, functional outcome, and intracranial bleeding. Circulation. 2007;115(17):2323-2330.

46. Boulay $\mathrm{G}$, et al. Modulation of $\mathrm{Ca}(2+)$ entry by polypeptides of the inositol 1,4, 5-trisphosphate receptor (IP3R) that bind transient receptor poten- 
tial (TRP): evidence for roles of TRP and IP3R in store depletion-activated $\mathrm{Ca}(2+)$ entry. Proc Natl Acad Sci U S A. 1999;96(26):14955-14960.

47. Beliveau E, Lessard V, Guillemette G. STIM1 positively regulates the $\mathrm{Ca}^{2+}$ release activity of the inositol 1,4,5-trisphosphate receptor in bovine aortic endothelial cells. PLoS One. 2014;9(12):e114718.

48. Patterson RL, Boehning D, Snyder SH. Inositol 1,4,5-trisphosphate receptors as signal integrators. Annu Rev Biochem. 2004;73:437-465.

49. Prole DL, Taylor CW. Inositol 1,4,5-trisphosphate receptors and their protein partners as signalling hubs. J Physiol. 2016;594(11):2849-2866.

50. Malkusch S, Endesfelder U, Mondry J, Gelleri M, Verveer PJ, Heilemann M. Coordinate-based colocalization analysis of single-molecule localization microscopy data. Histochem Cell Biol. 2012;137(1):1-10.

51. Ambily A, et al. The role of plasma membrane STIM1 and $\mathrm{Ca}(2+)$ entry in platelet aggregation. STIM1 binds to novel proteins in human platelets. Cell Signal. 2014;26(3):502-511.

52. Feske S. CRAC channels and disease - From human CRAC channelopathies and animal models to novel drugs. Cell Calcium. 2019;80:112-116.
53. Schwenk F, Baron U, Rajewsky K. A cre-transgenic mouse strain for the ubiquitous deletion of loxPflanked gene segments including deletion in germ cells. Nucleic Acids Res. 1995;23(24):5080-5081.

54. Tiedt R, Schomber T, Hao-Shen H, Skoda RC. Pf4-Cre transgenic mice allow the generation of lineage-restricted gene knockouts for studying megakaryocyte and platelet function in vivo. Blood.2007;109(4):1503-1506.

55. Winkler C, Denker K, Wortelkamp S, Sickmann A. Silver- and Coomassie-staining protocols: detection limits and compatibility with ESI MS. Electrophoresis. 2007;28(12):2095-2099.

56. Premsler T, Lewandrowski U, Sickmann A, Zahedi RP. Phosphoproteome analysis of the platelet plasma membrane. Methods Mol Biol. 2011;728:279-290.

57. Stritt S, et al. A gain-of-function variant in DIAPH1 causes dominant macrothrombocytopenia and hearing loss. Blood. 2016;127(23):2903-2914.

58. Stritt S, et al. Profilin 1-mediated cytoskeletal rearrangements regulate integrin function in mouse platelets. Blood Adv. 2018;2(9):1040-1045.

59. Aurbach K, Spindler M, Haining EJ, Bender M, Pleines I. Blood collection, platelet isolation and measurement of platelet count and size in mice-a practical guide. Platelets. 2019;30(6):698-707.

60. Pleines I, et al. Multiple alterations of platelet functions dominated by increased secretion in mice lacking Cdc42 in platelets. Blood. 2010;115(16):3364-3373.

61. Heemskerk JW, Feijge MA, Rietman E, Hornstra G. Rat platelets are deficient in internal $\mathrm{Ca}^{2+}$ release and require influx of extracellular $\mathrm{Ca}^{2+}$ for activation. FEBS Lett. 1991;284(2):223-226.

62. Schuhmann MK, et al. CD28 superagonist-mediated boost of regulatory $\mathrm{T}$ cells increases thromboinflammation and ischemic neurodegeneration during the acute phase of experimental stroke. JCereb Blood Flow Metab. 2015;35(1):6-10.

63. Richter KN, et al. Glyoxal as an alternative fixative to formaldehyde in immunostaining and super-resolution microscopy. EMBO J. 2018;37(1):139-159.

64. Nieswandt B, Bergmeier W, Rackebrandt K, Gessner JE, Zirngibl H. Identification of critical antigen-specific mechanisms in the development of immune thrombocytopenic purpura in mice. Blood.2000;96(7):2520-2527.

65. Wolter S, et al. rapidSTORM: accurate, fast opensource software for localization microscopy. Nat Methods. 2012;9(11):1040-1041. 